Supporting Information for

\title{
Unprecedented $\mathrm{CO}_{2}$-Promoted Aminochlorination of Olefins with Chloramine-T
}

Satoshi Minakata, * Yoshimi Yoneda, Yoji Oderaotoshi, and Mitsuo Komatsu* Department of Applied Chemistry, Graduate School of Engineering, Osaka University, Yamadaoka 2-1, Suita, Osaka 565-0871, Japan

\section{Table of Contents}

General Methods

Typical Procedure

Spectral Data

References

${ }^{1} \mathrm{H}$ - and ${ }^{13} \mathrm{C}-\mathrm{NMR}$ Spectra of Major Products S9-S38 


\section{Experimental}

General Methods. Melting points were determined on a Yanaco melting point apparatus and are uncorrected. Infrared spectra were obtained on a JASCO FT/IR-410 infrared spectrophotometer. ${ }^{1} \mathrm{H}$ and ${ }^{13} \mathrm{C}$ NMR spectra were recorded on a JEOL FT-NMR JNM EX 270 spectrometer $\left({ }^{1} \mathrm{H}\right.$ NMR, $270 \mathrm{MHz} ;{ }^{13} \mathrm{C}$ NMR, $\left.68 \mathrm{MHz}\right)$ using tetramethylsilane as an internal standard. Mass spectra were measured on a JEOL JMS-DX303HF mass spectrometer. Elemental analyses were performed at the Analytical Center, Faculty of Engineering, Osaka University. Products were purified by chromatography on silica gel BW-300 (Fuji Silysia Chemical Co.). Analytical thin-layer chromatography was performed on precoated silica gel glass plates (silica gel $60 \mathrm{~F}_{254}, 0.25 \mathrm{~mm}$ thickness) (Merck Co.). C Compounds were visualized by UV light or treatment with an ethanolic solution of phosphomolybdic acid followed by heating. Since the minor regioisomers $\mathbf{2 d}, \mathbf{1 g}, \mathbf{2 h}$ and $\mathbf{2 i}$ could not be isolated in pure form, the structures were determined by some spectral data, mainly ${ }^{1} \mathrm{H}-\mathrm{NMR}$ and Mass, in a form of mixture with the corresponding major regioisomers.

\section{Typical Procedure for Aminochlorination of Olefins with Chloramine-T under $\mathrm{CO}_{2}$}

A magnetic stirring bar, Chloramine-T (281 mg, $1 \mathrm{mmol})$, styrene (104 mg, $1 \mathrm{mmol})$, and benzene $(3 \mathrm{~mL})$ were placed in a $50-\mathrm{mL}$ stainless steel autoclave lined with a glass liner. The autoclave was closed, purged three times with carbon dioxide, pressurized with $10 \mathrm{~atm}$ of $\mathrm{CO}_{2}$ and then stirred at room temperature for $6 \mathrm{~h}$. After discharging excess $\mathrm{CO}_{2}$, aqueous $\mathrm{Na}_{2} \mathrm{~S}_{2} \mathrm{O}_{3}(0.5 \mathrm{M}, 20 \mathrm{~mL})$ was added to the reaction mixture. The solution was then extracted with $\mathrm{CH}_{2} \mathrm{Cl}_{2}(10 \mathrm{~mL} \times 3)$. The combined organic extracts were dried over $\mathrm{Na}_{2} \mathrm{SO}_{4}$ and concentrated to give the crude product. Purification by flash column chromatography (silica gel; $30 \%$ ethyl acetate in hexane) gave $\mathbf{2 a}(247 \mathrm{mg}, 80 \%)$. 


\section{1-Chloro-1-phenyl-2-(p-toluenesulfonamido)ethane (1a) ${ }^{1}$}

$\mathrm{Cl}$ Colorless solid (247 mg, 80\%); Mp: 95-96 ${ }^{\circ} \mathrm{C}$; IR $\left(\mathrm{KBr}, \mathrm{cm}^{-1}\right) 3265(\mathrm{NH})$, 1334, $1155(\mathrm{~S}=\mathrm{O}) ;{ }^{1} \mathrm{H}-\mathrm{NMR}\left(\mathrm{CDCl}_{3}, 270 \mathrm{MHz}\right) \delta 2.44\left(\mathrm{~s}, 3 \mathrm{H}, \mathrm{Ar}-\mathrm{C}_{3}\right)$, NHTs $3.36-3.53\left(\mathrm{~m}, 2 \mathrm{H}, \mathrm{C}_{2}-\mathrm{NH}\right), 4.87$ (dd, $\left.1 \mathrm{H}, J=5.9,7.1 \mathrm{~Hz}, \mathrm{C} \underline{H}-\mathrm{Cl}\right), 4.87$ (br t,

1a $\quad 1 \mathrm{H}, \mathrm{N} \underline{\mathrm{H}}, \mathrm{D}_{2} \mathrm{O}$ exchangeable), 7.11-7.43 (m, 7H, Ar$\left.\underline{\mathrm{H}}\right), 7.73(\mathrm{~d}, 2 \mathrm{H}, J=8.4 \mathrm{~Hz}$, $\operatorname{Ar} \underline{\mathrm{H}}) ;{ }^{13} \mathrm{C} \mathrm{NMR}\left(\mathrm{CDCl}_{3}, 68 \mathrm{MHz}\right) \delta$ 21.3, 50.0, 61.4, 126.6, 126.9, 128.4, 128.5, 129.4, 136.4, 137.6, 143.3; MS (CI, methane): $m / z$ (relative intensity, \%) $310\left([\mathrm{M}+1]^{+}, 28\right), 274\left([\mathrm{M}-\mathrm{Cl}]^{+}\right.$, 100); HRMS (CI, methane): $m / z$ Calcd for $\mathrm{C}_{15} \mathrm{H}_{17} \mathrm{ClNO}_{2} \mathrm{~S}(\mathrm{M}+\mathrm{H}) 310.0669$, found 310.0664; Anal. Calcd for $\mathrm{C}_{15} \mathrm{H}_{16} \mathrm{ClNO}_{2} \mathrm{~S}$ : C, 58.15; H, 5.21; N, 4.52. Found: C, 58.26; H, 5.06; N, 4.43.

\section{2-Chloro-1-phenyl-1-(p-toluenesulfonamido)ethane (2a)}

NHTs Colorless solid (40.3 mg, 13\%); Mp: 173-174 ${ }^{\circ} \mathrm{C}$; IR ( $\left.\mathrm{KBr}, \mathrm{cm}^{-1}\right) 3257(\mathrm{NH})$, 1313, $1161(\mathrm{~S}=\mathrm{O}) ;{ }^{1} \mathrm{H}-\mathrm{NMR}\left(\mathrm{CDCl}_{3}, 270 \mathrm{MHz}\right) \delta 2.39\left(\mathrm{~s}, 3 \mathrm{H}, \mathrm{Ar}-\mathrm{C}_{3}\right), 3.72(\mathrm{~d}$, $\left.\mathrm{Cl} 2 \mathrm{H}, J=5.9 \mathrm{~Hz}, \underline{\mathrm{C}}_{2}-\mathrm{Cl}\right), 4.56(\mathrm{t}, 1 \mathrm{H}, J=5.9 \mathrm{~Hz}, \underline{\mathrm{CH}}-\mathrm{NH}), 5.22(\mathrm{br} \mathrm{d}, 1 \mathrm{H}, \mathrm{N} \underline{\mathrm{H}}$,

2a $\quad \mathrm{D}_{2} \mathrm{O}$ exchangeable), 7.03-7.30 (m, 7H, Ar $\left.\underline{\mathrm{H}}\right), 7.73(\mathrm{~d}, 2 \mathrm{H}, J=8.4 \mathrm{~Hz}, \operatorname{Ar} \underline{\mathrm{H}}) ;{ }^{13} \mathrm{C}$ NMR $\left(\mathrm{CDCl}_{3}, 68 \mathrm{MHz}\right) \delta$ 21.6, 47.9, 58.4, 126.7, 127.1, 128.2, 128.5, 129.4, 136.8, 143.4; MS (CI, isobutane): $m / z$ (relative intensity, \%) $310\left([\mathrm{M}+1]^{+}, 100\right), 274$ ([M-Cl $\left.]^{+}, 24\right)$; HRMS (CI, isobutane): $m / z$ Calcd for $\mathrm{C}_{15} \mathrm{H}_{17} \mathrm{ClNO}_{2} \mathrm{~S}(\mathrm{M}+\mathrm{H}) 310.0669$, found 310.0674; Anal. Calcd for $\mathrm{C}_{15} \mathrm{H}_{16} \mathrm{ClNO}_{2} \mathrm{~S}$ : C, 58.15; H, 5.21; N, 4.52. Found: C, 58.12; H, 4.96; N, 4.43.

\section{1-Chloro-1-(4-nitrophenyl)-2-( $p$-toluenesulfonamido)ethane (1b)}<smiles>O=[N+]([O-])c1ccc(C(Cl)CN[AsH3])cc1</smiles>

$1 \mathrm{~b}$

Yellow solid (251 mg, 71\%); Mp: $111-113{ }^{\circ} \mathrm{C}$; IR $\left(\mathrm{KBr}, \mathrm{cm}^{-1}\right) 3278$ $(\mathrm{NH}), 1522,1346\left(\mathrm{NO}_{2}\right), 1163(\mathrm{~S}=\mathrm{O}) ;{ }^{1} \mathrm{H}-\mathrm{NMR}\left(\mathrm{CDCl}_{3}, 270 \mathrm{MHz}\right) \delta$ $2.43\left(\mathrm{~s}, 3 \mathrm{H}, \mathrm{Ar}-\underline{\mathrm{C}}_{3}\right), 3.38-3.54\left(\mathrm{~m}, 2 \mathrm{H}, \mathrm{C}_{2}-\mathrm{NH}\right), 5.00(\mathrm{t}, 1 \mathrm{H}, J=6.8$ $\mathrm{Hz}, \mathrm{C} \underline{\mathrm{H}}-\mathrm{Cl}$ ), 5.20 (t, 1H, $J=6.5 \mathrm{~Hz}, \underline{\mathrm{N}}, \mathrm{D}_{2} \mathrm{O}$ exchangeable) 7.29 (d, $2 \mathrm{H}, J=8.1 \mathrm{~Hz}, \operatorname{Ar} \underline{\mathrm{H}}), 7.49$ (d, 2H, $J=8.6 \mathrm{~Hz}, \operatorname{Ar} \underline{\mathrm{H}}), 7.68$ (d, 2H, $J=$

$8.1 \mathrm{~Hz}, \operatorname{Ar} \underline{\mathrm{H}}), 8.15(\mathrm{~d}, 2 \mathrm{H}, J=8.6 \mathrm{~Hz}, \operatorname{Ar} \underline{\mathrm{H}}) ;{ }^{13} \mathrm{C} \mathrm{NMR}\left(\mathrm{CDCl}_{3}, 68 \mathrm{MHz}\right) \delta 21.4,49.8,59.6$, 123.7, 126.6, 128.3, 129.6, 136.1, 143.8, 144.6, 147.5; MS (CI, isobutane): $\mathrm{m} / z$ (relative intensity, \%) 355 ([M+1] $\left.]^{+}, 81\right), 319$ ([M-Cl] $\left.]^{+}, 100\right)$; HRMS (CI, isobutane): $m / z$ Calcd for $\mathrm{C}_{15} \mathrm{H}_{16} \mathrm{ClN}_{2} \mathrm{O}_{4} \mathrm{~S}(\mathrm{M}+\mathrm{H})$ 355.0520, found 355.0523; Anal. Calcd for $\mathrm{C}_{15} \mathrm{H}_{15} \mathrm{ClN}_{2} \mathrm{O}_{4} \mathrm{~S}$ : C, 50.78; H, 4.26; N, 7.90. Found: C, 50.77; H, 4.12; N, 7.74. 


\section{1-Chloro-1-(4-chlorophenyl)-2-(p-toluenesulfonamido)ethane (1c)}<smiles>SNCC(Cl)c1ccc(Cl)cc1</smiles>

$1 \mathrm{c}$

Colorless solid (254 mg, 74\%); Mp: 100-102 ${ }^{\circ} \mathrm{C}$; IR $\left(\mathrm{KBr}, \mathrm{cm}^{-1}\right) 3282$ $(\mathrm{NH}), 1325,1153(\mathrm{~S}=\mathrm{O}) ;{ }^{1} \mathrm{H}-\mathrm{NMR}\left(\mathrm{CDCl}_{3}, 270 \mathrm{MHz}\right) \delta 2.44(\mathrm{~s}, 3 \mathrm{H}$, Ar- $\left.\underline{\mathrm{H}}_{3}\right), 3.33-3.50\left(\mathrm{~m}, 2 \mathrm{H}, \underline{\mathrm{CH}}_{2} \mathrm{NH}\right), 4.81$ (br t, $1 \mathrm{H}, \mathrm{N} \underline{\mathrm{H}}, \mathrm{D}_{2} \mathrm{O}$ exchangeable), 4.87 (dd, $1 \mathrm{H}, J=6.2,7.8 \mathrm{~Hz}, \mathrm{C} \underline{\mathrm{H}}-\mathrm{Cl}$ ), 7.20-7.35 (m, $6 \mathrm{H}, \mathrm{Ar} \underline{\mathrm{H}}), 7.71(\mathrm{~d}, 2 \mathrm{H}, J=8.4 \mathrm{~Hz}, \mathrm{Ar} \underline{\mathrm{H}}) ;{ }^{13} \mathrm{C} \mathrm{NMR}\left(\mathrm{CDCl}_{3}, 68 \mathrm{MHz}\right) \oint$ 21.5, 50.0, 60.5, 126.7, 128.5, 128.7, 129.6, 134.4, 136.2, 137.3, 143.5; MS (CI, isobutane): $\mathrm{m} / \mathrm{z}$ (relative intensity, \%) $344\left([\mathrm{M}+1]^{+}, 7\right), 308\left([\mathrm{M}-\mathrm{Cl}]^{+}, 100\right)$; HRMS (CI, isobutane): $\mathrm{m} / \mathrm{z}$ Calcd for $\mathrm{C}_{15} \mathrm{H}_{16} \mathrm{Cl}_{2} \mathrm{NO}_{2} \mathrm{~S}(\mathrm{M}+\mathrm{H})$ 344.0280, found 344.0285; Anal. Calcd for $\mathrm{C}_{15} \mathrm{H}_{15} \mathrm{Cl}_{2} \mathrm{NO}_{2} \mathrm{~S}$ : C, 52.33; H, 4.39; N, 4.07. Found: C, 52.37; H, 4.27; N, 4.04.

\section{1-Chloro-1-(4-methylphenyl)-2-(p-toluenesulfonamido)ethane (1d)}<smiles>Cc1ccc(C(Cl)CNS)cc1</smiles>

$1 d$

Colorless solid (174 mg, 54\%); Mp: $119-121{ }^{\circ} \mathrm{C}$; IR (KBr, cm $\left.{ }^{-1}\right) 3245$ $(\mathrm{NH}), 1325,1159(\mathrm{~S}=\mathrm{O})$; ${ }^{1} \mathrm{H}-\mathrm{NMR}\left(\mathrm{CDCl}_{3}, 270 \mathrm{MHz}\right) \delta 2.34(\mathrm{~s}, 3 \mathrm{H}$, Ar- $\left.\underline{\mathrm{H}}_{3}\right), 2.44$ (s, 3H, Ar- $\left.\underline{\mathrm{H}}_{3}\right), 3.37-3.49$ (m, 2H, $\left.\underline{\mathrm{C}}_{2}-\mathrm{NH}\right), 4.77$ (br t, $1 \mathrm{H}, \mathrm{N} \underline{\mathrm{H}}, \mathrm{D}_{2} \mathrm{O}$ exchangeable), $4.83(\mathrm{dd}, 1 \mathrm{H}, J=6.5,8.1 \mathrm{~Hz}, \mathrm{C} \underline{\mathrm{H}}-\mathrm{Cl})$, 6.97-7.25 (m, 4H, Arth), 7.32 (d, $2 \mathrm{H}, J=8.4 \mathrm{~Hz}, \operatorname{Ar} \underline{\mathrm{H}}), 7.72(\mathrm{~d}, 2 \mathrm{H}, J=8.4 \mathrm{~Hz}, \operatorname{Ar} \underline{\mathrm{H}}) ;{ }^{13} \mathrm{C}$ $\mathrm{NMR}\left(\mathrm{CDCl}_{3}, 68 \mathrm{MHz}\right) \delta .21 .1,21.5,50.2,61.5,126.9,129.4,129.6,134.7,136.7,138.8$, 143.5; MS (CI, isobutane): $m / z$ (relative intensity, \%): $324\left([\mathrm{M}+1]^{+}, 4\right), 288\left([\mathrm{M}-\mathrm{Cl}]^{+}, 100\right)$; HRMS (CI, isobutane): $\mathrm{m} / z$ Calcd for $\mathrm{C}_{16} \mathrm{H}_{19} \mathrm{ClNO}_{2} \mathrm{~S}(\mathrm{M}+\mathrm{H})$ 324.0826, found 324.0816; Anal.: Calcd for $\mathrm{C}_{16} \mathrm{H}_{18} \mathrm{ClNO}_{2} \mathrm{~S}$ : C, 59.34; H, 5.60; N, 4.33. Found: C, 59.32; H, 5.52; N, 4.28 .

\section{2-Chloro-1-(4-methoxyphenyl)-1-(p-toluenesulfonamido)ethane (2e)}

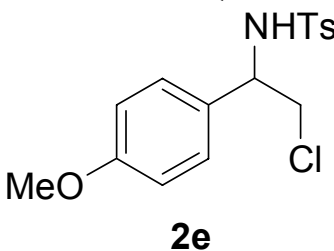

Colorless solid (257 mg, 76\%); Mp: $120-121^{\circ} \mathrm{C}$; IR (KBr, cm $\left.{ }^{-1}\right) 3274$ $(\mathrm{NH}), 1325(\mathrm{~S}=\mathrm{O}), 1251(\mathrm{MeO}), 1162(\mathrm{~S}=\mathrm{O}) ;{ }^{1} \mathrm{H}-\mathrm{NMR}\left(\mathrm{CDCl}_{3}, 270\right.$ $\mathrm{MHz}) \delta 2.40\left(\mathrm{~s}, 3 \mathrm{H}, \mathrm{Ar}-\underline{\mathrm{H}}_{3}\right), 3.66-3.71\left(\mathrm{~m}, 2 \mathrm{H}, \underline{\mathrm{C}}_{2}-\mathrm{Cl}\right), 3.77(\mathrm{~s}, 3 \mathrm{H}$, $\left.\mathrm{C}_{3}-\mathrm{O}\right), 4.50$ (t, $\left.1 \mathrm{H}, J=5.9 \mathrm{~Hz}, \mathrm{C} \underline{\mathrm{H}}-\mathrm{NH}\right), 5.15$ (br d, $1 \mathrm{H}, \mathrm{N} \underline{\mathrm{H}}, \mathrm{D}_{2} \mathrm{O}$ exchangeable), 6.76 (d, 2H, $J=8.6 \mathrm{~Hz}, \operatorname{Ar} \underline{\mathrm{H}}), 7.03$ (d, 2H, $J=8.6 \mathrm{~Hz}, \operatorname{Ar} \underline{\mathrm{H}}), 7.21$ (d, 2H, $J=$ $8.1 \mathrm{~Hz}, \operatorname{Ar} \underline{\mathrm{H}}), 7.62(\mathrm{~d}, 2 \mathrm{H}, J=8.1 \mathrm{~Hz}, \operatorname{Ar} \underline{\mathrm{H}}) ;{ }^{13} \mathrm{C} \mathrm{NMR}\left(\mathrm{CDCl}_{3}, 68 \mathrm{MHz}\right) \delta 21.5,47.7,55.1$, 58.0, 113.7, 126.9, 127.9, 129.1, 129.3, 136.7, 143.2, 159.1; MS (EI): $\mathrm{m} / z$ (relative intensity, \%) $339\left([\mathrm{M}]^{+}, 1\right), 290\left(\left[\mathrm{M}-\mathrm{CH}_{2} \mathrm{Cl}\right]^{+}, 100\right)$; HRMS (EI): $m / z$ Calcd for $\mathrm{C}_{16} \mathrm{H}_{18} \mathrm{ClNO}_{3} \mathrm{~S}(\mathrm{M})$ 339.0696, found 339.0699; Anal. Calcd for $\mathrm{C}_{16} \mathrm{H}_{18} \mathrm{ClNO}_{3} \mathrm{~S}$ : C, 56.55; $\mathrm{H}$, 5.34; N, 4.12. Found: C, 56.34; H, 5.17; N, 4.05. 


\section{1-Chloro-1-phenyl-2-(p-toluenesulfonamido)propane (1f) ${ }^{2}$}

$\mathrm{Cl} \quad$ Colorless solid (168 mg, $52 \%$ for the reaction from trans- $\beta$-methylstyrene); $\mathrm{Ph}$ Me Mp: $72-74{ }^{\circ} \mathrm{C}$; IR $\left(\mathrm{KBr}, \mathrm{cm}^{-1}\right) 3264(\mathrm{NH}), 1336,1165(\mathrm{~S}=\mathrm{O}) ;{ }^{1} \mathrm{H}-\mathrm{NMR}\left(\mathrm{CDCl}_{3}\right.$, $\overline{\mathrm{N}} \mathrm{HTs} 270 \mathrm{MHz}) \delta 1.02\left(\mathrm{~d}, 3 \mathrm{H}, J=6.8 \mathrm{~Hz}, \mathrm{C}_{3}-\mathrm{CH}\right), 2.43\left(\mathrm{~s}, 3 \mathrm{H}, \mathrm{Ar}-\underline{\mathrm{H}}_{3}\right), 3.70-3.82$ $1 f(\mathrm{~m}, 1 \mathrm{H}, \mathrm{C} \underline{\mathrm{H}}-\mathrm{NH}), 4.91\left(\mathrm{~d}, 1 \mathrm{H}, J=9.2 \mathrm{~Hz}, \mathrm{~N} \underline{\mathrm{H}}, \mathrm{D}_{2} \mathrm{O}\right.$ exchangeable $), 5.02(\mathrm{~d}, 1 \mathrm{H}$, $J=6.2 \mathrm{~Hz}, \mathrm{C} \underline{\mathrm{H}}-\mathrm{Cl}), 7.24-7.36(\mathrm{~m}, 7 \mathrm{H}, \mathrm{Ar} \underline{\mathrm{H}}), 7.77$ (d, $2 \mathrm{H}, J=8.4 \mathrm{~Hz}, \mathrm{Ar} \underline{\mathrm{H}}) ;{ }^{13} \mathrm{C} \mathrm{NMR}\left(\mathrm{CDCl}_{3}\right.$, $68 \mathrm{MHz}) \delta 15.2,21.5,55.3,68.0,127.0,127.2,128.4,128.5,129.8,137.4,137.9 .143 .6$; MS (CI, isobutane): $m / z$ (relative intensity, \%) $324\left([\mathrm{M}+1]^{+}, 20\right), 288\left([\mathrm{M}-\mathrm{Cl}]^{+}, 100\right)$; HRMS (CI, isobutane): $\mathrm{m} / z$ Calcd for $\mathrm{C}_{16} \mathrm{H}_{19} \mathrm{ClNO}_{2} \mathrm{~S}(\mathrm{M}+\mathrm{H})$ 324.0826, found 324.0818; Anal.: Calcd for $\mathrm{C}_{16} \mathrm{H}_{18} \mathrm{ClNO}_{2} \mathrm{~S}$ : C, 59.34; H, 5.60; N, 4.33. Found: C, 59.29; H, 5.47; N, 4.28.

\section{2-Chloro-2-phenyl-1-(p-toluenesulfonamido)propane (1h)}<smiles>CC(Cl)(CN[18F])c1ccccc1</smiles>

Colorless solid (177 mg, 55\%); Mp: 78-80 ${ }^{\circ} \mathrm{C}$; IR (KBr, cm $\left.{ }^{-1}\right) 3275(\mathrm{NH})$, 1325, $1161(\mathrm{~S}=\mathrm{O}) ;{ }^{1} \mathrm{H}-\mathrm{NMR}\left(\mathrm{CDCl}_{3}, 270 \mathrm{MHz}\right) \delta 1.98\left(\mathrm{~s}, 3 \mathrm{H}, \mathrm{C}_{3}-\mathrm{CCl}\right)$, $2.43\left(\mathrm{~s}, 3 \mathrm{H}, \mathrm{Ar}-\underline{\mathrm{C}}_{3}\right), 3.37-3.52\left(\mathrm{~m}, 2 \mathrm{H}, \underline{\mathrm{CH}}_{2} \mathrm{NH}\right), 4.71(\mathrm{t}, 1 \mathrm{H}, J=6.2 \mathrm{~Hz}$, $\mathrm{N} \underline{\mathrm{H}}, \mathrm{D}_{2} \mathrm{O}$ exchangeable), 7.26-7.37 (m, 5H, Ar$\left.\underline{\mathrm{H}}\right), 7.47$ (d, 2H, $J=8.4 \mathrm{~Hz}$, $\operatorname{Ar} \underline{\mathrm{H}}), 7.68(\mathrm{~d}, 2 \mathrm{H}, J=8.4 \mathrm{~Hz}, \operatorname{Ar} \underline{\mathrm{H}}) ;{ }^{13} \mathrm{C} \mathrm{NMR}\left(\mathrm{CDCl}_{3}, 68 \mathrm{MHz}\right) \delta .21 .6$, 29.0, 55.4, 71.8, 126.0, 126.8, 128.1, 128.4, 129.6, 136.6, 141.6, 143.4; MS (CI, isobutane): $\mathrm{m} / z$ (relative intensity, \%): $324\left([\mathrm{M}+1]^{+}, 3\right), 288\left([\mathrm{M}-\mathrm{Cl}]^{+}, 100\right)$; HRMS (CI, isobutane): $m / z$ Calcd for $\mathrm{C}_{16} \mathrm{H}_{19} \mathrm{ClNO}_{2} \mathrm{~S}(\mathrm{M}+\mathrm{H})$ 324.0826, found 324.0824; Anal.: Calcd for $\mathrm{C}_{16} \mathrm{H}_{18} \mathrm{ClNO}_{2} \mathrm{~S}$ : C, 59.34; H, 5.60; N, 4.33. Found: C, 59.14; H, 5.53; N, 4.39.

\section{1-Chloro-2-(p-toluenesulfonamido)indane (1i)}

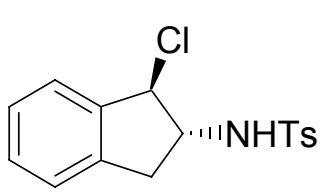

$1 \mathrm{i}$

Brown solid (202 mg, 63\%); Mp: 106-109 ${ }^{\circ} \mathrm{C}$; IR ( $\left.\mathrm{KBr}, \mathrm{cm}^{-1}\right) 3265(\mathrm{NH})$, 1329, $1161(\mathrm{~S}=\mathrm{O}) ;{ }^{1} \mathrm{H}-\mathrm{NMR}\left(\mathrm{CDCl}_{3}, 270 \mathrm{MHz}\right) \delta 2.45\left(\mathrm{~s}, 3 \mathrm{H}, \mathrm{Ar}-\underline{\mathrm{H}}_{3}\right)$, $2.74\left(\mathrm{dd}, 1 \mathrm{H}, J=4.9,16.2 \mathrm{~Hz}, \mathrm{C}_{2}-\mathrm{CH}-\mathrm{NH}\right), 3.39$ (dd, $1 \mathrm{H}, J=7.0,16.2$ $\left.\mathrm{Hz}, \underline{\mathrm{C}}_{2}-\mathrm{CH}-\mathrm{NH}\right), 4.03-4.30$ (m, 1H, $\left.\underline{\mathrm{C}}-\mathrm{NH}\right), 4.99$ (d, $1 \mathrm{H}, J=7.6 \mathrm{~Hz}$, $\mathrm{N} \underline{\mathrm{H}}, \mathrm{D}_{2} \mathrm{O}$ exchangeable), $5.08(\mathrm{~d}, 1 \mathrm{H}, J=4.6 \mathrm{~Hz}, \mathrm{C} \underline{\mathrm{H}}-\mathrm{Cl}), 7.06-7.35$ (m, $6 \mathrm{H}, \operatorname{Ar} \underline{\mathrm{H}}), 7.79$ (d, 2H, $J=8.4 \mathrm{~Hz}, \mathrm{Ar} \underline{\mathrm{H}}) ;{ }^{13} \mathrm{C} \mathrm{NMR}\left(\mathrm{CDCl}_{3}, 68 \mathrm{MHz}\right) \delta$ 21.8, 37.8, 63.3, 65.3, $124.9,125.3,127.2,127.7,129.4,129.8,136.7,139.3,139.4,143.8$; MS (CI, isobutane): $\mathrm{m} / z$ (relative intensity, \%): $322\left([\mathrm{M}+1]^{+}, 7\right), 286\left([\mathrm{M}-\mathrm{Cl}]^{+}, 100\right)$; HRMS (CI, isobutane): $\mathrm{m} / \mathrm{z}$ Calcd for $\mathrm{C}_{16} \mathrm{H}_{17} \mathrm{ClNO}_{2} \mathrm{~S}(\mathrm{M}+\mathrm{H})$ 322.0669, found 322.0659; Anal.: Calcd for $\mathrm{C}_{16} \mathrm{H}_{16} \mathrm{ClNO}_{2} \mathrm{~S}$ : C, 59.71; H, 5.01; N, 4.35. Found: C, 59.81; H, 4.96; N, 4.15. 


\section{1-Chloro-2-(p-toluenesulfonamido)-3,4-dihydronaphthalene (1j)}<smiles>Cl[C@H]1CCc2ccccc2[C@H]1N[I-]</smiles>

$1 \mathrm{j}$

Brown solid (178 mg, 53\%); Mp: 106-108 C; IR (KBr, cm $\left.{ }^{-1}\right) 3280(\mathrm{NH})$, 1329, $1155(\mathrm{~S}=\mathrm{O}) ;{ }^{1} \mathrm{H}-\mathrm{NMR}\left(\mathrm{CDCl}_{3}, 270 \mathrm{MHz}\right) \delta$ 1.66-1.77 (m, $1 \mathrm{H}$, $\left.\mathrm{CH}_{2}-\underline{\mathrm{C}}_{2}-\mathrm{CH}-\mathrm{NH}\right), 2.38-2.51\left(\mathrm{~m}, 1 \mathrm{H}, \mathrm{CH}_{2}-\underline{\mathrm{C}}_{2}-\mathrm{CH}-\mathrm{NH}\right), 2.47$ (s, 3H, Ar- $\left.\underline{H}_{3}\right), 2.73-2.93\left(\mathrm{~m}, 2 \mathrm{H}, \underline{\mathrm{C}}_{2}-\mathrm{CH}_{2}-\mathrm{CH}-\mathrm{NH}\right), 3.82-3.89(\mathrm{~m}, 1 \mathrm{H}$,

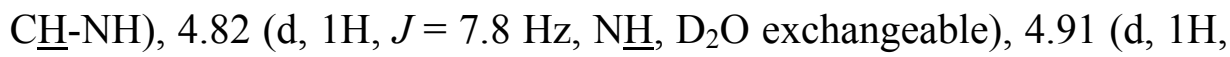
$J=4.1 \mathrm{~Hz}, \mathrm{C} \underline{\mathrm{H}}-\mathrm{Cl}), 7.26-7.37$ (m, 4H, Ar$\underline{\mathrm{H}}), 7.47$ (d, 2H, $J=8.4 \mathrm{~Hz}, \operatorname{Ar} \underline{\mathrm{H}}), 7.68$ (d, 2H, $J=$ $8.4 \mathrm{~Hz}, \operatorname{Ar} \underline{\mathrm{H}}) ;{ }^{13} \mathrm{C} \mathrm{NMR}\left(\mathrm{CDCl}_{3}, 68 \mathrm{MHz}\right) \oint 21.7,23.0,23.8,54.5,58.5,126.6,126.8,128.6$, 128.9, 129.7, 130.9, 132.6, 134.8, 136.9, 143.5; MS (CI, isobutane): $m / z$ (relative intensity, \%) $336\left([\mathrm{M}+1]^{+}, 4\right), 300\left([\mathrm{M}-\mathrm{Cl}]^{+}, 100\right)$; HRMS (CI, isobutane): $m / z$ Calcd for $\mathrm{C}_{17} \mathrm{H}_{19} \mathrm{ClNO}_{2} \mathrm{~S}$ $(\mathrm{M}+\mathrm{H}) 336.0826$, found 336.0827

\section{2-Chloro-1-(p-toluenesulfonamido)-3,4-dihydronaphthalene (2j)}<smiles>FC(F)(F)N[C@@H]1c2ccccc2CC[C@@H]1Cl</smiles>

$2 \mathrm{j}$

Brown solid (50 mg, 15\%); Mp: 164-165 ${ }^{\circ} \mathrm{C}$; IR (KBr, $\left.\mathrm{cm}^{-1}\right) 3277(\mathrm{NH})$, 1331, $1152(\mathrm{~S}=\mathrm{O}) ;{ }^{1} \mathrm{H}-\mathrm{NMR}\left(\mathrm{CDCl}_{3}, 270 \mathrm{MHz}\right) \delta 2.07-2.13(\mathrm{~m}, 1 \mathrm{H}$, $\left.\mathrm{CH}_{2}-\underline{\mathrm{C}}_{2}-\mathrm{CH}-\mathrm{Cl}\right), 2.31-2.45\left(\mathrm{~m}, 1 \mathrm{H}, \mathrm{CH}_{2}-\underline{\mathrm{C}}_{2}-\mathrm{CH}-\mathrm{Cl}\right), 2.50 \quad(\mathrm{~s}, 3 \mathrm{H}$, $\left.\operatorname{Ar}-\underline{\mathrm{C}}_{3}\right), \quad 2.71-2.80 \quad\left(\mathrm{~m}, \quad 1 \mathrm{H}, \quad \mathrm{C}_{2}-\mathrm{CH}_{2}-\mathrm{CH}-\mathrm{Cl}\right), \quad 3.00-3.13 \quad(\mathrm{~m}, \quad 1 \mathrm{H}$, $\left.\mathrm{C}_{2}-\mathrm{CH}_{2}-\mathrm{CH}-\mathrm{Cl}\right), 4.38(\mathrm{~m}, 1 \mathrm{H}, \mathrm{C} \underline{\mathrm{H}}-\mathrm{Cl}), 4.55-4.59$ (m, 1H, C$\left.-\underline{\mathrm{H}}-\mathrm{NH}\right), 4.59$

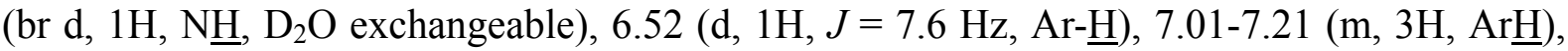
7.40 (d, 2H, $J=8.4 \mathrm{~Hz}, \mathrm{Ar} \underline{\mathrm{H}}), 7.84(\mathrm{~d}, 2 \mathrm{H}, J=8.4 \mathrm{~Hz}, \mathrm{Ar} \underline{\mathrm{H}}) ;{ }^{13} \mathrm{C} \mathrm{NMR}\left(\mathrm{CDCl}_{3}, 68 \mathrm{MHz}\right) \delta$ 21.8, 23.6, 24.7, 56.8, 58.4, 126.7, 127.3, 128.2, 129.0, 129.6, 129.8, 131.2, 135.7, 136.7, 143.9; MS (CI, isobutane): $m / z$ (relative intensity, \%) $336\left([\mathrm{M}+1]^{+}, 100\right), 300\left([\mathrm{M}-\mathrm{Cl}]^{+}, 82\right)$, HRMS (CI, isobutane): $\mathrm{m} / z$ Calcd for $\mathrm{C}_{17} \mathrm{H}_{19} \mathrm{ClNO}_{2} \mathrm{~S}(\mathrm{M}+\mathrm{H})$ 336.0826, found 336.0828; Anal. Calcd for $\mathrm{C}_{17} \mathrm{H}_{18} \mathrm{ClNO}_{2} \mathrm{~S}$ : C, 60.80; H, 5.40; N, 4.17. Found: C, 60.99; H, 5.43; N, 4.10.

3-Chloro-4-(p-toluenesulfonamido)hexane (1k) Stereochemistry was determined by the conversion of product $\mathbf{1 k}$ to a known aziridine. ${ }^{3}$

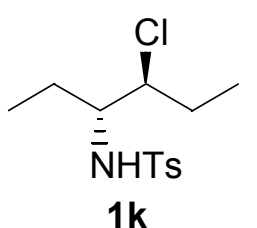

Colorless solid (81 mg, 28\%); Mp: 95-97 ${ }^{\circ} \mathrm{C}$; IR (KBr, $\left.\mathrm{cm}^{-1}\right) 3278(\mathrm{NH})$, 1323, $1168(\mathrm{~S}=\mathrm{O}) ;{ }^{1} \mathrm{H}-\mathrm{NMR}\left(\mathrm{CDCl}_{3}, 270 \mathrm{MHz}\right) \delta 0.79(\mathrm{t}, 3 \mathrm{H}, J=7.4 \mathrm{~Hz}$, $\left.\mathrm{C}_{3}-\mathrm{CH}_{2}\right), 0.94\left(\mathrm{t}, 3 \mathrm{H}, J=7.4 \mathrm{~Hz}, \underline{\mathrm{CH}}_{3}-\mathrm{CH}_{2}\right), 1.36-1.71(\mathrm{~m}, 4 \mathrm{H}$, $\left.\mathrm{CH}_{3}-\underline{\mathrm{H}}_{2}-\mathrm{CH}-\mathrm{CH}-\underline{\mathrm{C}}_{2}-\mathrm{CH}_{3}\right), 2.43\left(\mathrm{~s}, 3 \mathrm{H}, \quad \mathrm{Ar}-\underline{\mathrm{C}}_{3}\right), 3.27-3.37(\mathrm{~m}, 1 \mathrm{H}$,

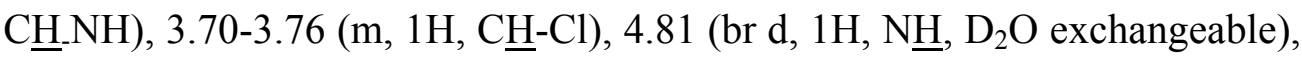
$7.30(\mathrm{~d}, 2 \mathrm{H}, J=8.4 \mathrm{~Hz}, \mathrm{Ar} \underline{\mathrm{H}}), 7.76(\mathrm{~d}, 2 \mathrm{H}, J=8.4 \mathrm{~Hz}, \mathrm{Ar} \underline{\mathrm{H}}) ;{ }^{13} \mathrm{C} \mathrm{NMR}\left(\mathrm{CDCl}_{3}, 68 \mathrm{MHz}\right)$ $\delta$ 10.2, 11.6.21.6, 21.8, 28.4, 59.0, 69.2, 126.8, 129.5, 137.9, 143.3; MS (CI, isobutane): $\mathrm{m} / \mathrm{z}$ (relative intensity, \%) $290\left([\mathrm{M}+1]^{+}, 100\right), 254$ ([M-Cl $\left.]^{+}, 8\right)$; HRMS (CI, isobutane): $m / z$ Calcd for $\mathrm{C}_{13} \mathrm{H}_{21} \mathrm{ClNO}_{2} \mathrm{~S}(\mathrm{M}+\mathrm{H})$ 290.0982, found 290.0989; Anal. Calcd for $\mathrm{C}_{13} \mathrm{H}_{20} \mathrm{ClNO}_{2} \mathrm{~S}$ : C, 53.87; H, 6.96; N, 4.83. Found: C, 53.98; H, 6.78; N, 4.78. 


\section{1-Chloro-2-(p-toluenesulfonamido)cyclohexane (11) ${ }^{4}$}

$\overbrace{1 / \mathrm{NHTS}}^{\mathrm{Cl}}$

11

Colorless solid (77 mg, 27\%); Mp: 99-101 ${ }^{\circ} \mathrm{C}$; IR (KBr, $\left.\mathrm{cm}^{-1}\right) 3255(\mathrm{NH})$, 1334, $1155(\mathrm{~S}=\mathrm{O}) ;{ }^{1} \mathrm{H}-\mathrm{NMR}\left(\mathrm{CDCl}_{3}, 270 \mathrm{MHz}\right) \delta 1.23-1.31(\mathrm{~m}, 3 \mathrm{H}$, $\left.\mathrm{C}_{2}-\mathrm{C} \underline{H} \mathrm{H}\right), 1.54-1.72$ (m, 3H, $\left.\underline{\mathrm{C}}_{2}-\mathrm{C} \underline{\mathrm{H}}\right), 1.36-1.71$ (m, 2H, $\mathrm{CH}_{2}-\mathrm{CH} \underline{\mathrm{H}}$,

$\left.\mathrm{CH}_{2}-\mathrm{CH} \underline{\mathrm{H}}\right), 2.43$ (s, 3H, Ar- $\underline{\mathrm{H}}_{3}$ ), 3.27-3.37 (m, 1H, $\left.\mathrm{C} \underline{\mathrm{H}}-\mathrm{NH}\right), 3.70-3.76$ (m, 1H, $\mathrm{C} \underline{\mathrm{H}}-\mathrm{Cl}$ ), 4.81 (br d, 1H, $\underline{\mathrm{H}}, \mathrm{D}_{2} \mathrm{O}$ exchangeable), 7.30 (d, 2H, J=8.4 Hz, Ar프), 7.76 (d, $2 \mathrm{H}, J=8.4 \mathrm{~Hz}, \mathrm{Ar} \underline{\mathrm{H}}) ;{ }^{13} \mathrm{C} \mathrm{NMR}\left(\mathrm{CDCl}_{3}, 68 \mathrm{MHz}\right) \delta 21.6,23.4_{2} .24 .3,32.4,34.9,58.6,62.1$, 127.1, 129.4, 137.0, 143.3; MS (CI, isobutane): $m / z$ (relative intensity, \%) $288\left([\mathrm{M}+1]^{+}, 100\right)$, 252 ([M-Cl] $\left.]^{+}, 23\right)$; HRMS (CI, isobutane): $\mathrm{m} / z$ Calcd for $\mathrm{C}_{13} \mathrm{H}_{19} \mathrm{ClNO}_{2} \mathrm{~S}(\mathrm{M}+\mathrm{H})$ 288.0826, found 288.0829; Anal. Calcd for $\mathrm{C}_{13} \mathrm{H}_{18} \mathrm{ClNO}_{2} \mathrm{~S}$ : C, 54.25; H, 6.30; N, 4.87. Found: C, 54.50; $\mathrm{H}, 6.05 ; \mathrm{N}, 4.82$.

\section{2-Chloro-1-n-butoxy-1-(p-toluenesulfonamido)ethane (1m)}

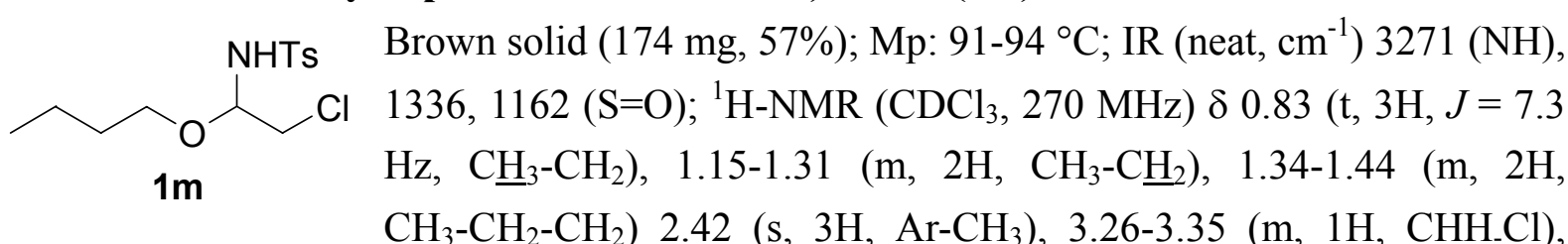
3.41-3.55 (m, 3H, $\left.\underline{\mathrm{H}}_{2} \mathrm{O}, \mathrm{C} \underline{\mathrm{H}}-\mathrm{Cl}\right), 4.74-4.88$ (m, $\left.1 \mathrm{H}, \mathrm{C} \underline{\mathrm{H}}-\mathrm{NH}\right), 5.76$ (d, $1 \mathrm{H}, J=9.5 \mathrm{~Hz}, \mathrm{~N} \underline{\mathrm{H}}$, $\mathrm{D}_{2} \mathrm{O}$ exchangeable), 7.30 (d, $\left.2 \mathrm{H}, J=8.4 \mathrm{~Hz}, \mathrm{Ar} \underline{\mathrm{H}}\right), 7.78$ (d, 2H, $\left.J=8.4 \mathrm{~Hz}, \operatorname{Ar} \underline{\mathrm{H}}\right) ;{ }^{13} \mathrm{C} \mathrm{NMR}$ $\left(\mathrm{CDCl}_{3}, 68 \mathrm{MHz}\right) \delta 13.7,19.0,21.5,31.1,46.0,68.2,83.1,126.6,129.5,137.9,143.5 . \mathrm{MS}$ (FAB): $m / z$ (relative intensity, \%) 304 ([M-1] $\left.]^{+}, 84\right)$; HRMS (FAB): $m / z$ Calcd for $\mathrm{C}_{13} \mathrm{H}_{19} \mathrm{ClNO}_{3} \mathrm{~S}(\mathrm{M}-\mathrm{H})$ 304.0772, found 304.0770.

\section{1-Chloro-4-(p-toluenesulfonamido)-2-cyclooctene (1n)}

NHTs Colorless solid (157 mg, 70\%); Mp: 93-94 ${ }^{\circ} \mathrm{C}$; IR (KBr, $\left.\mathrm{cm}^{-1}\right) 3259(\mathrm{NH}), 1328$, $1161(\mathrm{~S}=\mathrm{O}) ;{ }^{1} \mathrm{H}-\mathrm{NMR}\left(\mathrm{CDCl}_{3}, 270 \mathrm{MHz}\right) \delta$ 1.31-1.88 (m, 6H, $\left.\left(\mathrm{C}_{2}\right)_{3}-\mathrm{CH}_{2}\right)$, 1.95-2.19 (m, 2H, $\left.\left(\mathrm{CH}_{2}\right)_{3}-\mathrm{C}_{2}\right), 2.42\left(\mathrm{~s}, 3 \mathrm{H}, \mathrm{Ar} \mathrm{C}_{-} \underline{H}_{3}\right), 4.57-4.73(\mathrm{~m}, 2 \mathrm{H}$, C$-\mathrm{NH}, \mathrm{C} \underline{\mathrm{H}}-\mathrm{Cl}), 4.64$ (br, $1 \mathrm{H}, \mathrm{N} \underline{\mathrm{H}}, \mathrm{D}_{2} \mathrm{O}$ exchangeable), 5.18 (dd, $1 \mathrm{H}, J=6.8$, $11.9 \mathrm{~Hz}, \mathrm{C} \underline{\mathrm{H}}-\mathrm{CHCl}), 5.65$ (dd, $1 \mathrm{H}, J=6.5,11.9 \mathrm{~Hz}, \mathrm{C} \underline{\mathrm{H}}-\mathrm{CHNH}), 7.30$ (d, 1H, $J$ $=8.1 \mathrm{~Hz}, \operatorname{Ar} \underline{\mathrm{H}}), 7.78(\mathrm{~d}, 2 \mathrm{H}, J=8.1 \mathrm{~Hz}, \operatorname{Ar} \underline{\mathrm{H}}) ;{ }^{13} \mathrm{C} \mathrm{NMR}\left(\mathrm{CDCl}_{3}, 68 \mathrm{MHz}\right) \delta$ 21.5, 22.5, 23.4, 34.5, 37.2, 51.1, 57.6, 127.1, 129.4, 130.2, 132.0, 137.1, 143.1; MS (CI, isobutane): $m / z$ (relative intensity, \%) $314\left([\mathrm{M}+1]^{+}, 3\right), 278\left([\mathrm{M}-\mathrm{Cl}]^{+}, 100\right)$, HRMS (CI, isobutane): $\mathrm{m} / z$ Calcd for $\mathrm{C}_{15} \mathrm{H}_{21} \mathrm{ClNO}_{2} \mathrm{~S}(\mathrm{M}+\mathrm{H})$ 314.0982, found 314.0991; Anal. Calcd for $\mathrm{C}_{15} \mathrm{H}_{20} \mathrm{ClNO}_{2} \mathrm{~S}$ : C, 57.40; H, 6.42; N, 4.46. Found: C, 57.29; H, 6.25; N, 4.42. 


\section{References}

1. Yadav, J. S.; Reddy, B. V. S.; Kumar, G. M. Synlett 2001. 9. 1417.

2. Flores-Parra, A.; Suárez-Moreno, P.; Sánchez-Ruíz, S. A.; Tlahuextl, M.; Jaen-Gaspar, J.; Tlahuext, H.; Salas-Coronado, R.; Cruz, A.; Nöth, H.; Contreras, R. Tetrahedron Asymmetry 1998. 9. 1661.

3. Jeong, J. U.; Taao, B.; Sagasser, I.; Henniges, H.; Sharpless, K. B. J. Am. Chem. Soc. 1998. 120. 6844 .

4. Pandey, M. K.; Bisai, A.; Singh, V. K. Tetrahedron Lett. 2004. 45. 9661. 


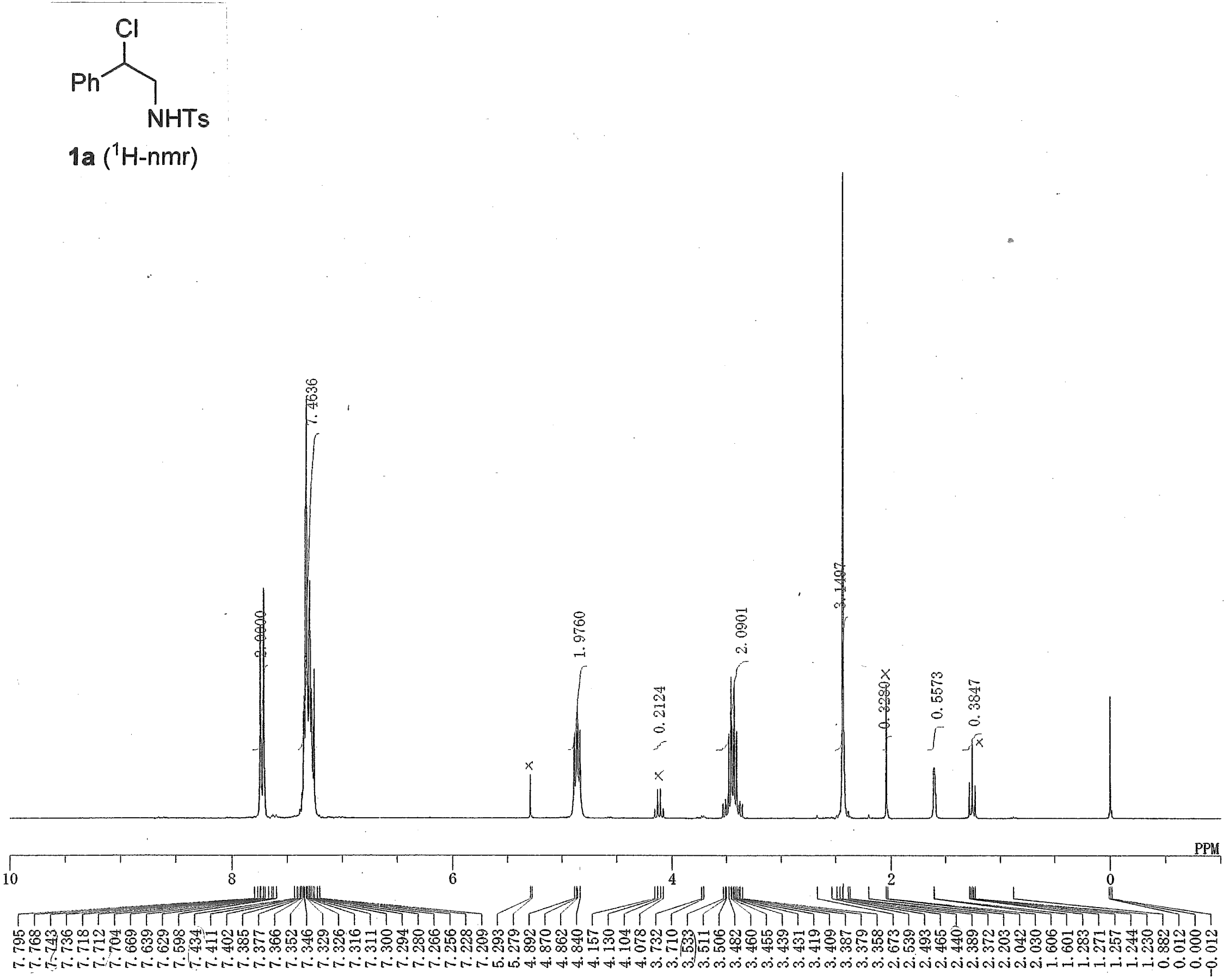



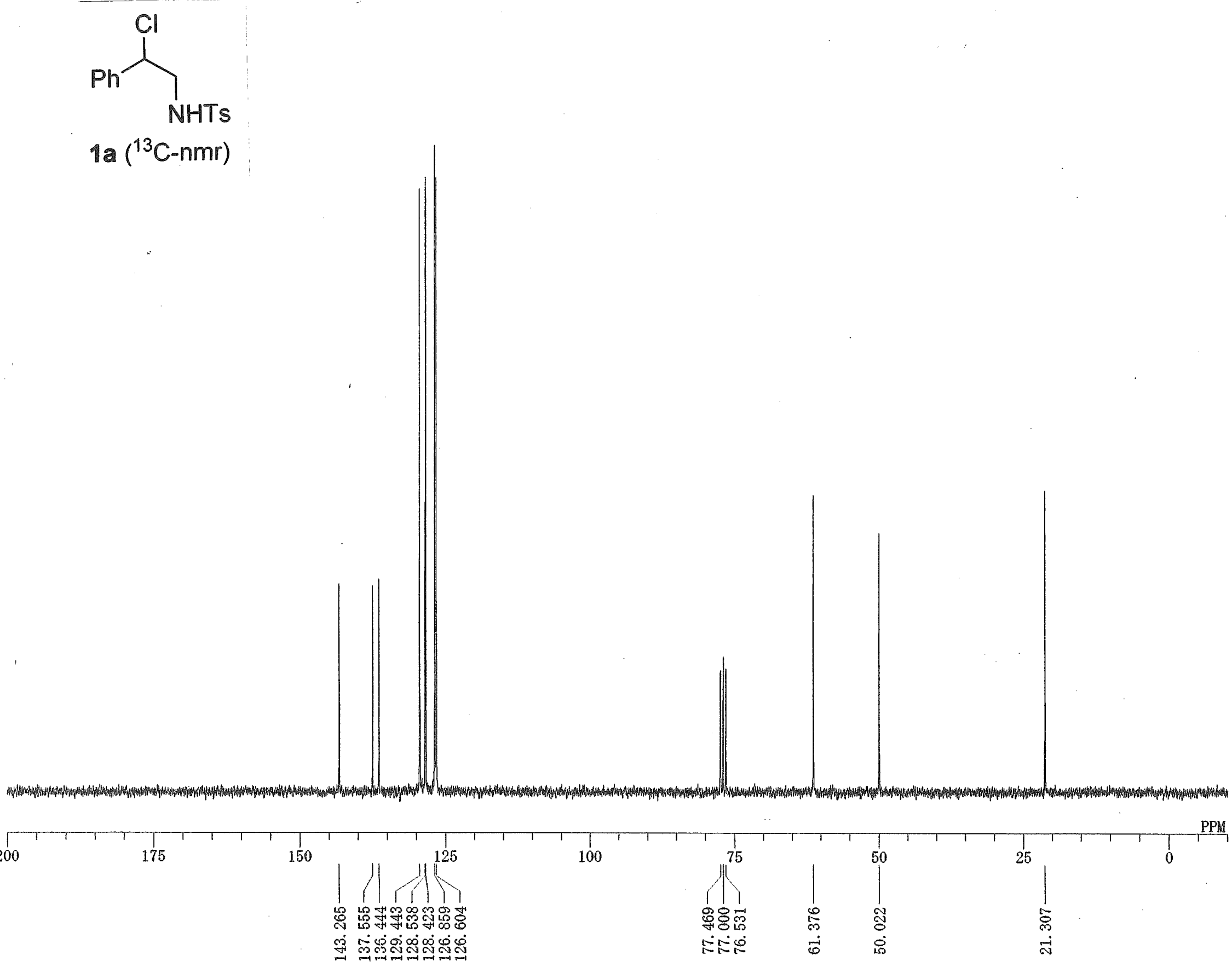


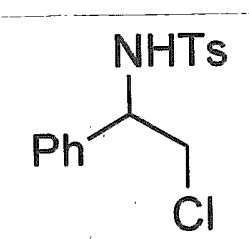

$2 \mathrm{a}\left({ }^{1} \mathrm{H}-\mathrm{nmr}\right)$
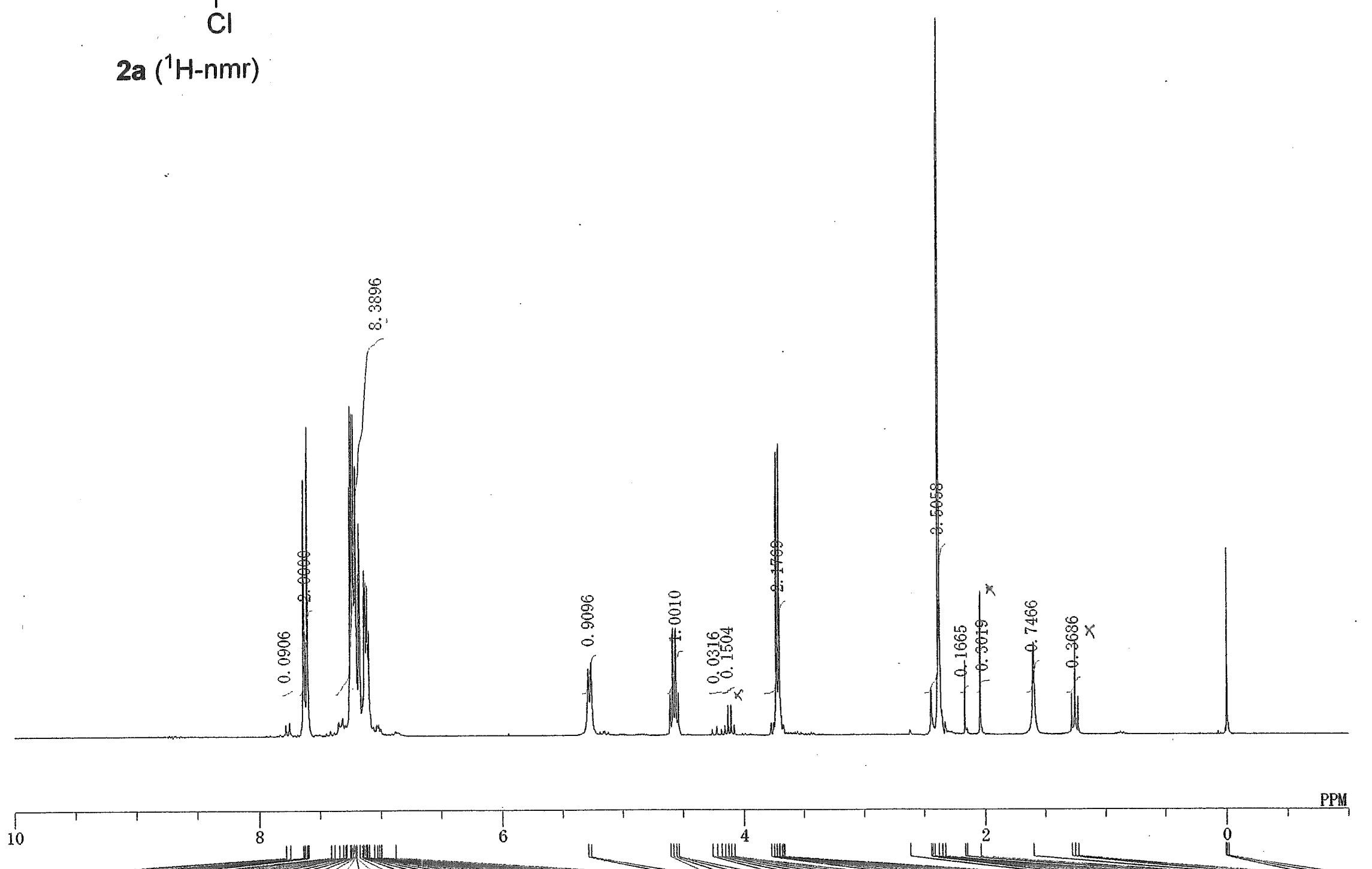

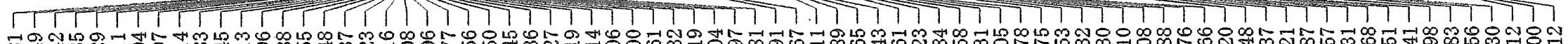

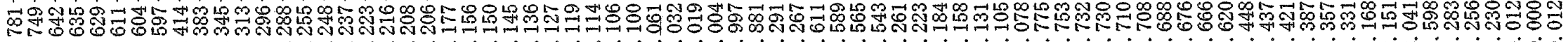

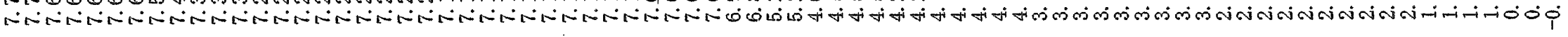




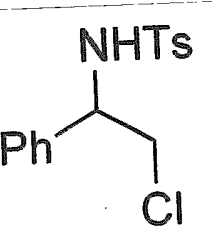

$2 a\left({ }^{13} \mathrm{C}-\mathrm{nm} r\right)$

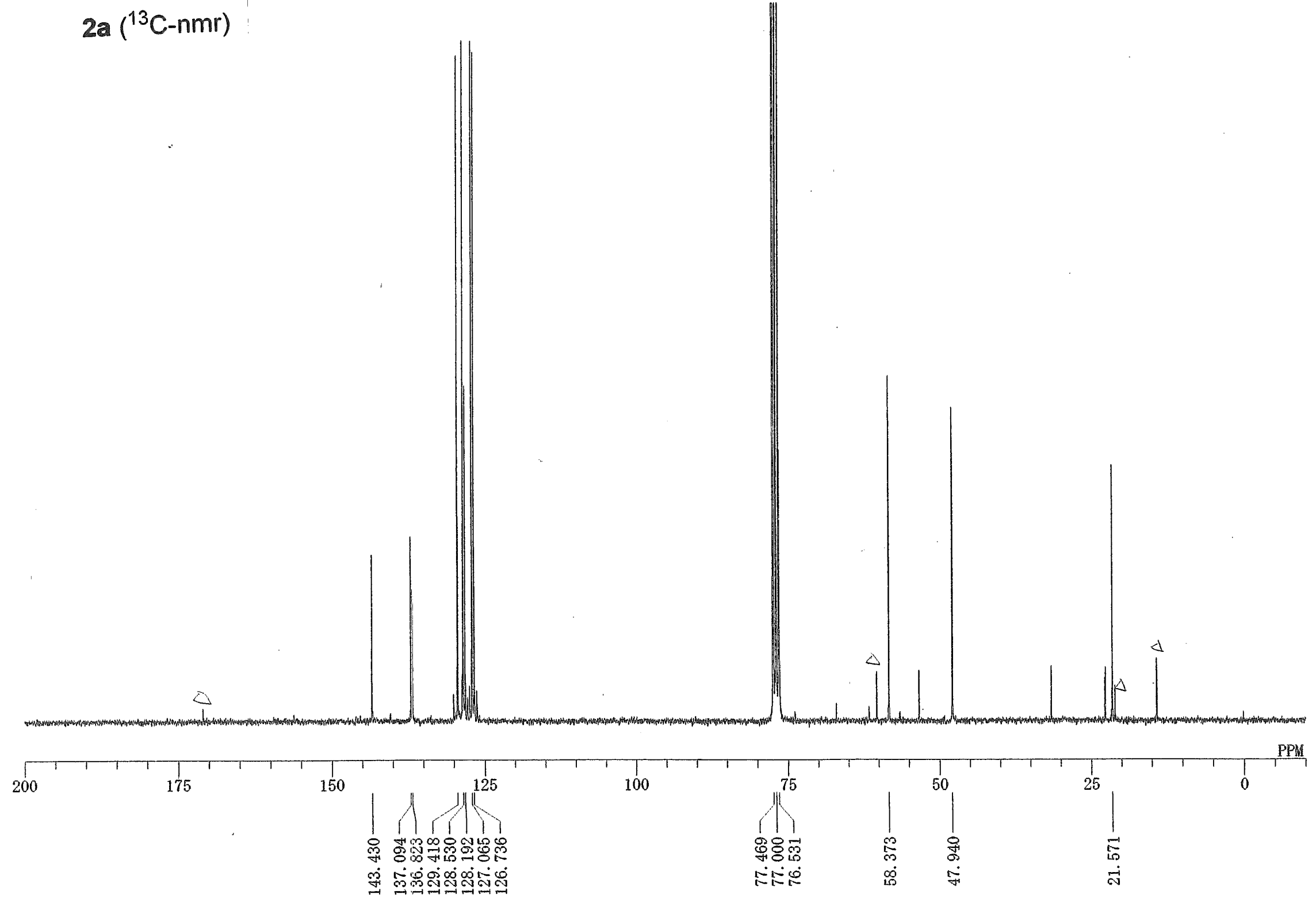

$\triangle$ FLOAC 

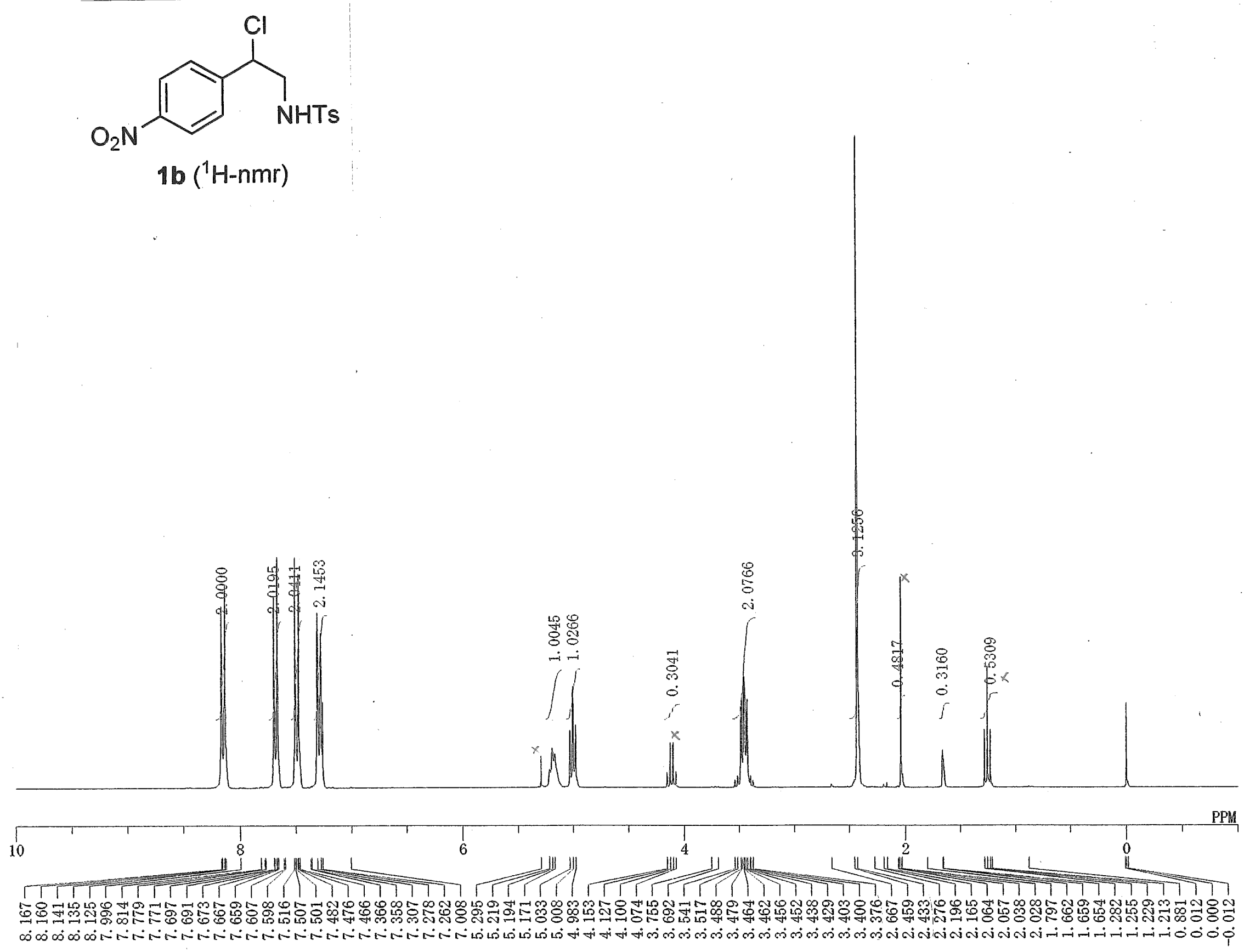

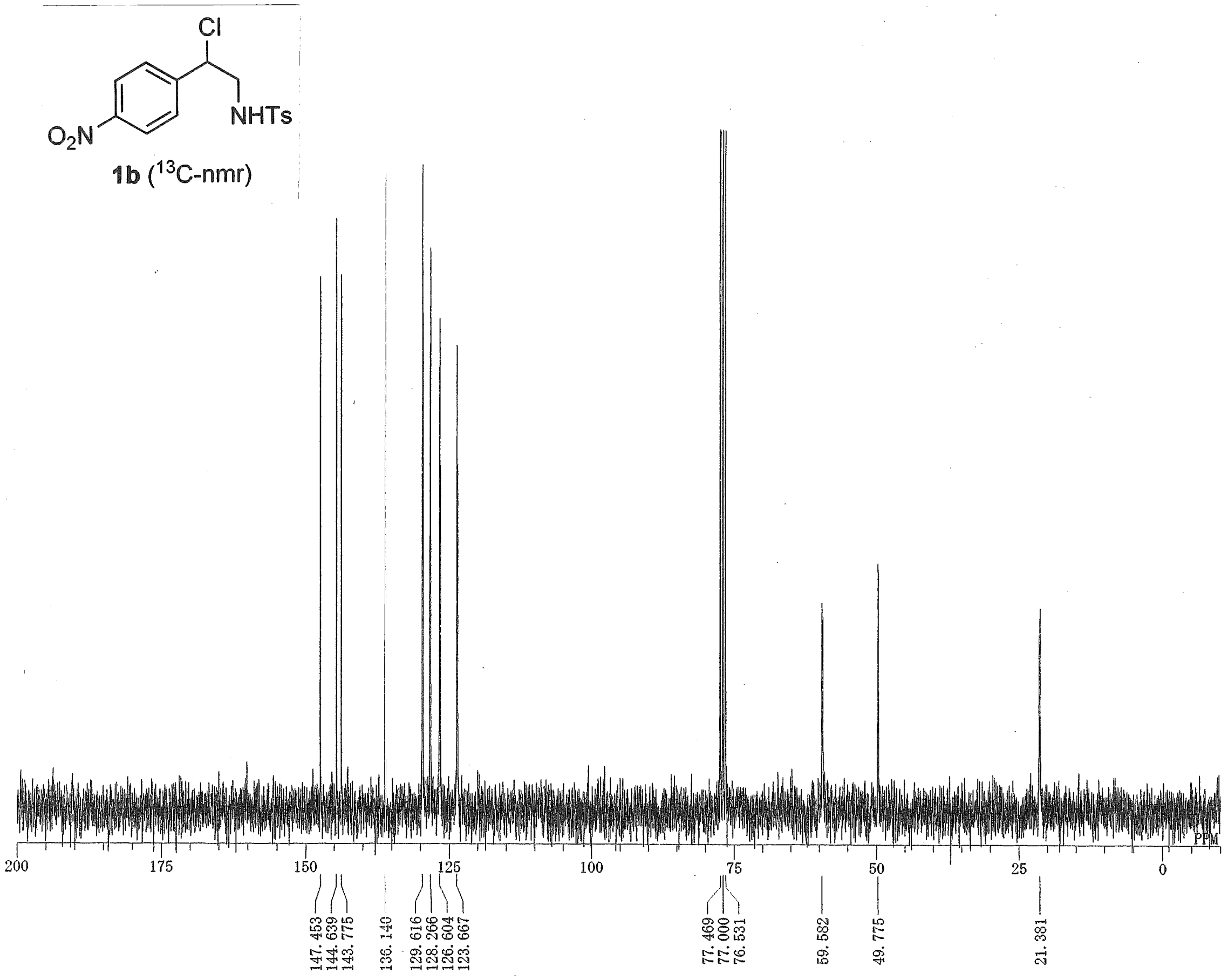

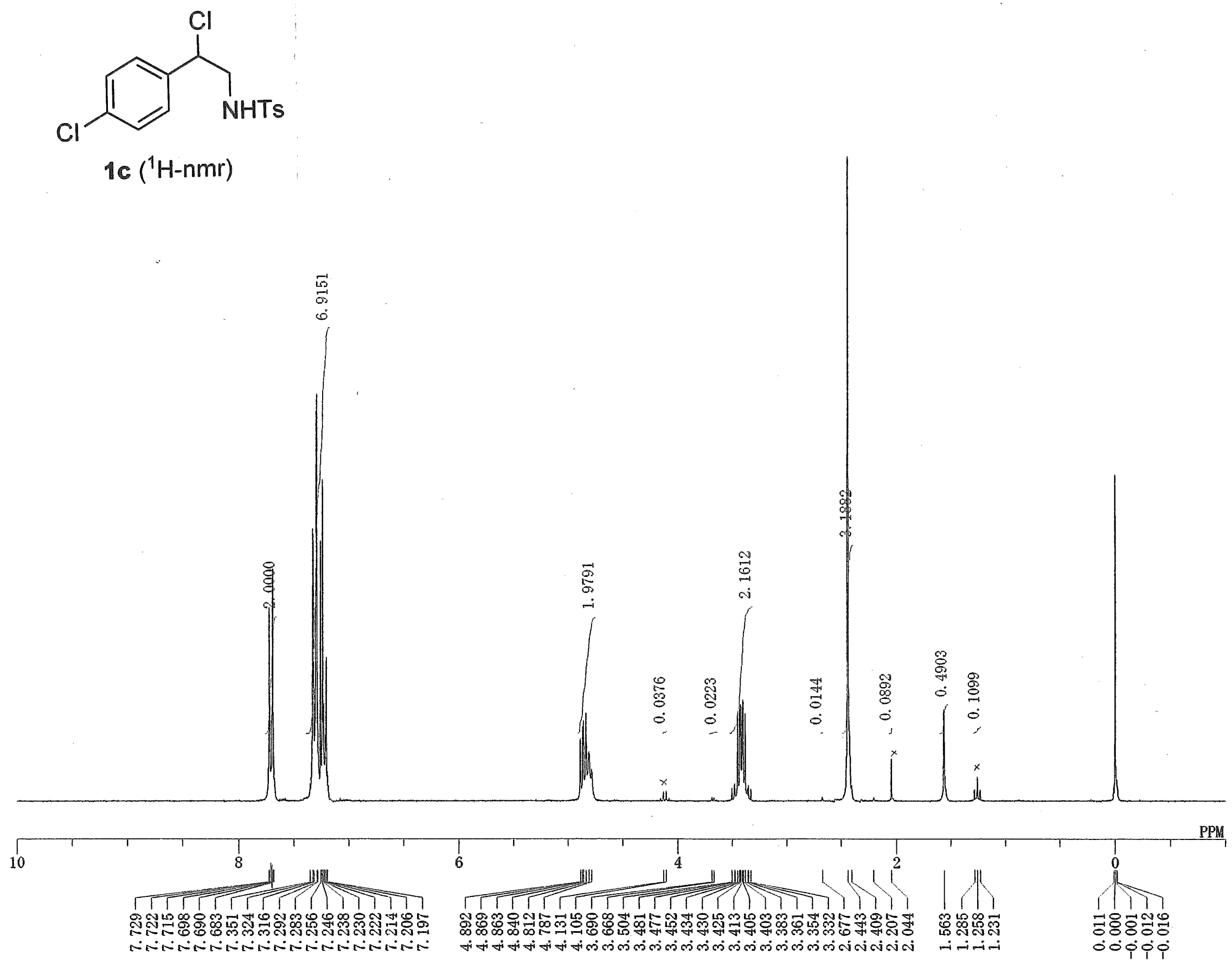


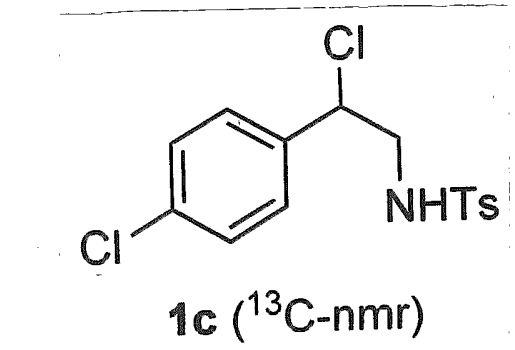

Hed.t.

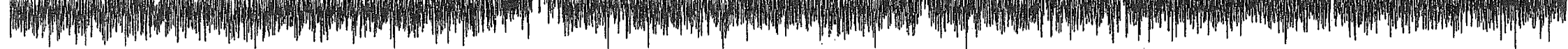

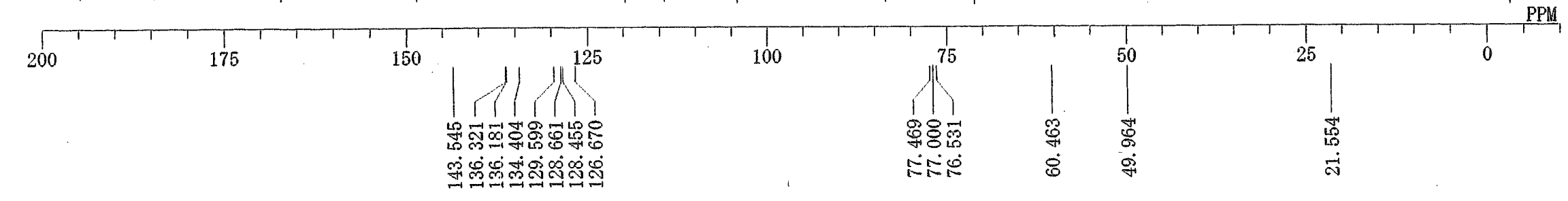




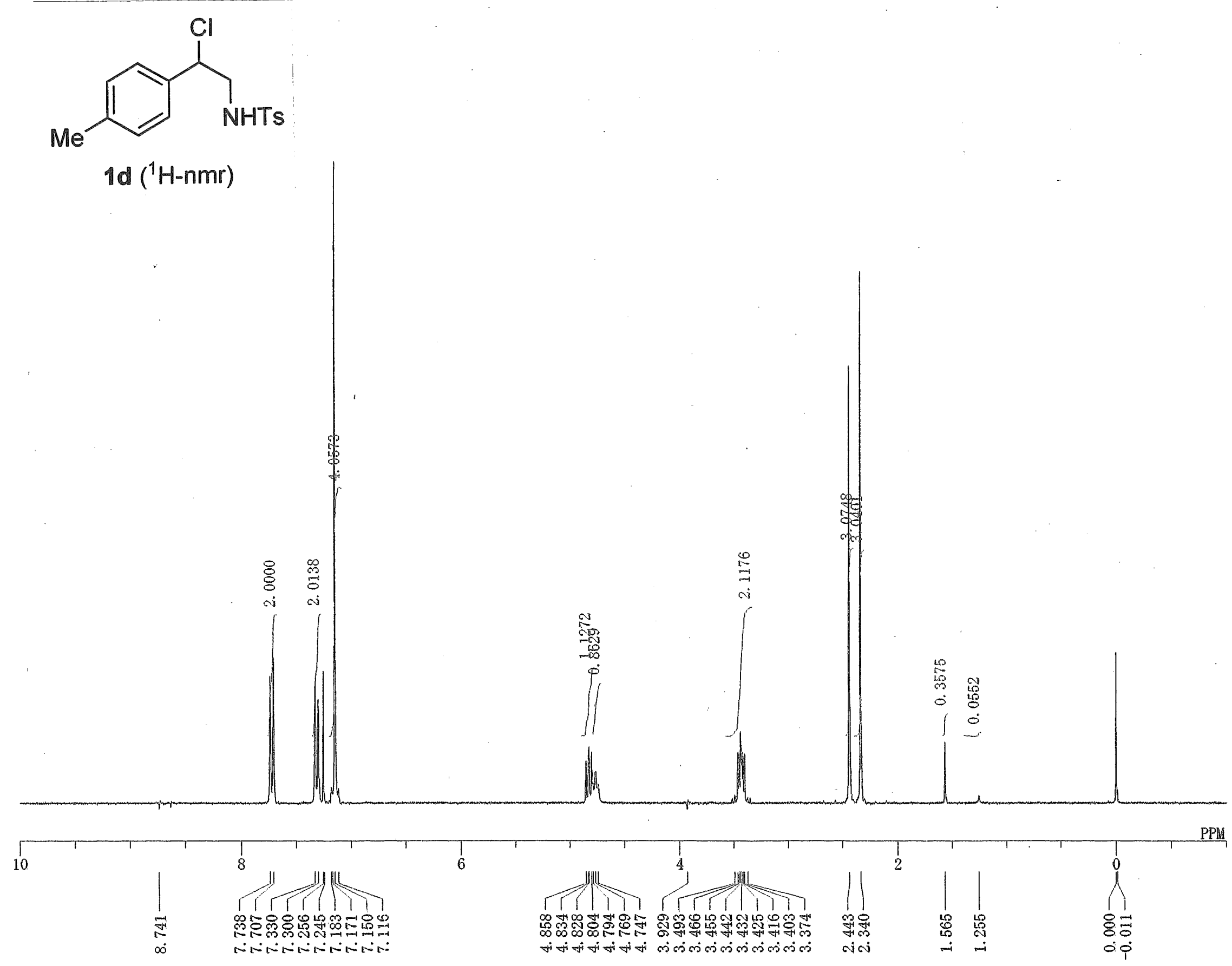




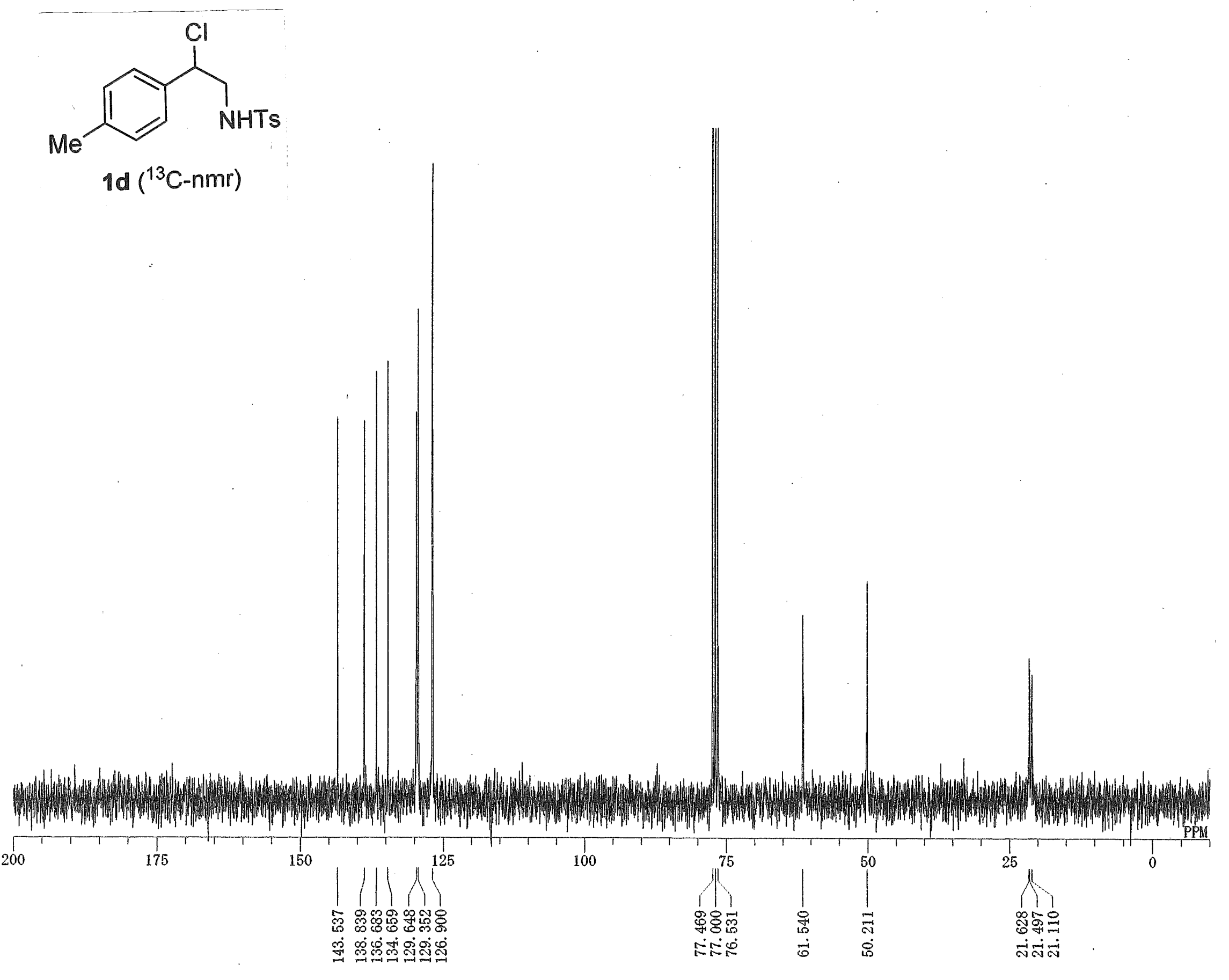




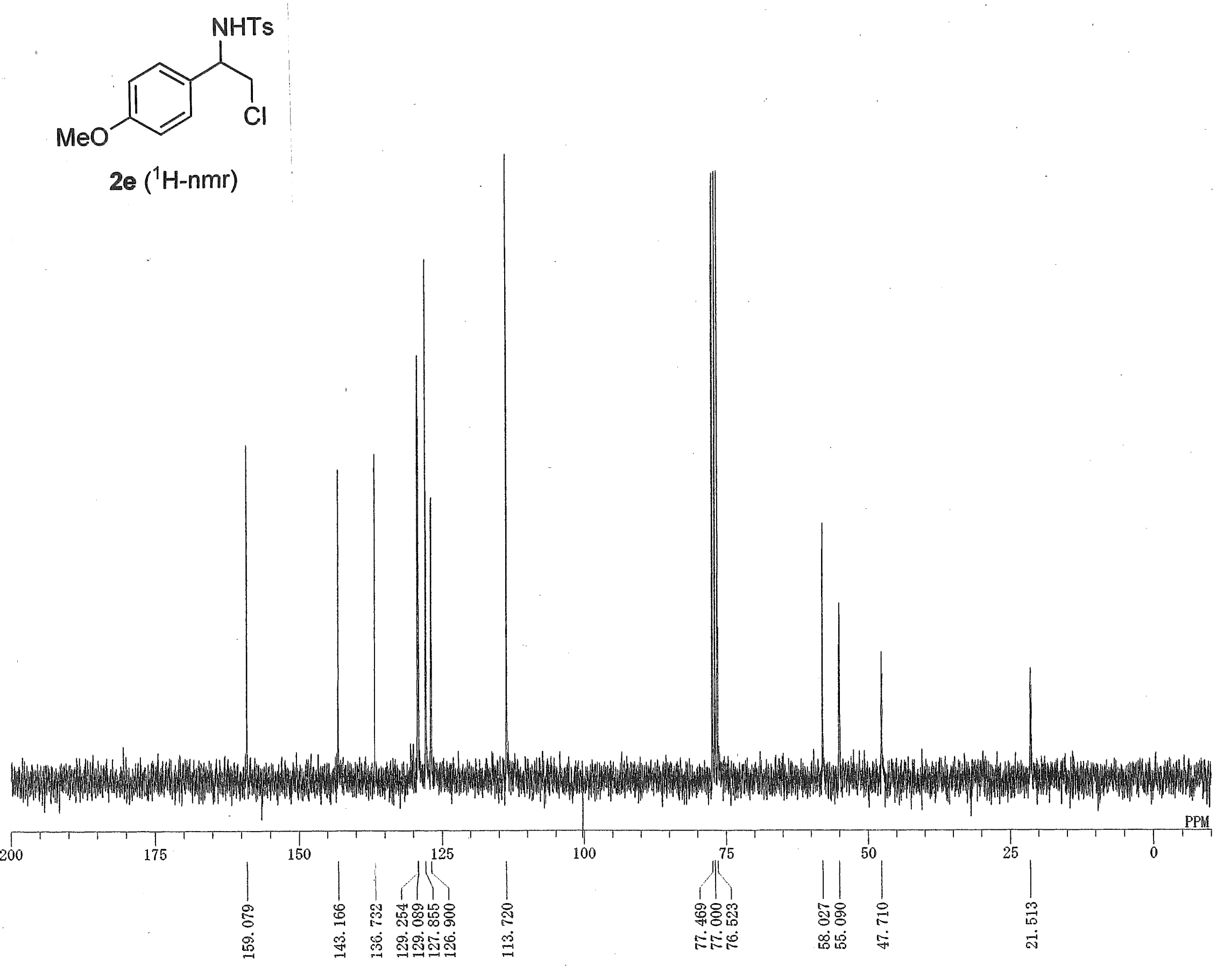




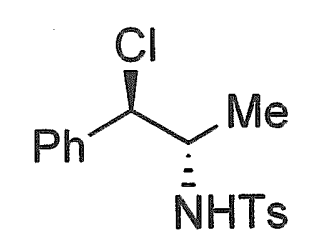

$1 \mathrm{f}\left({ }^{1} \mathrm{H}-\mathrm{nmr}\right)$

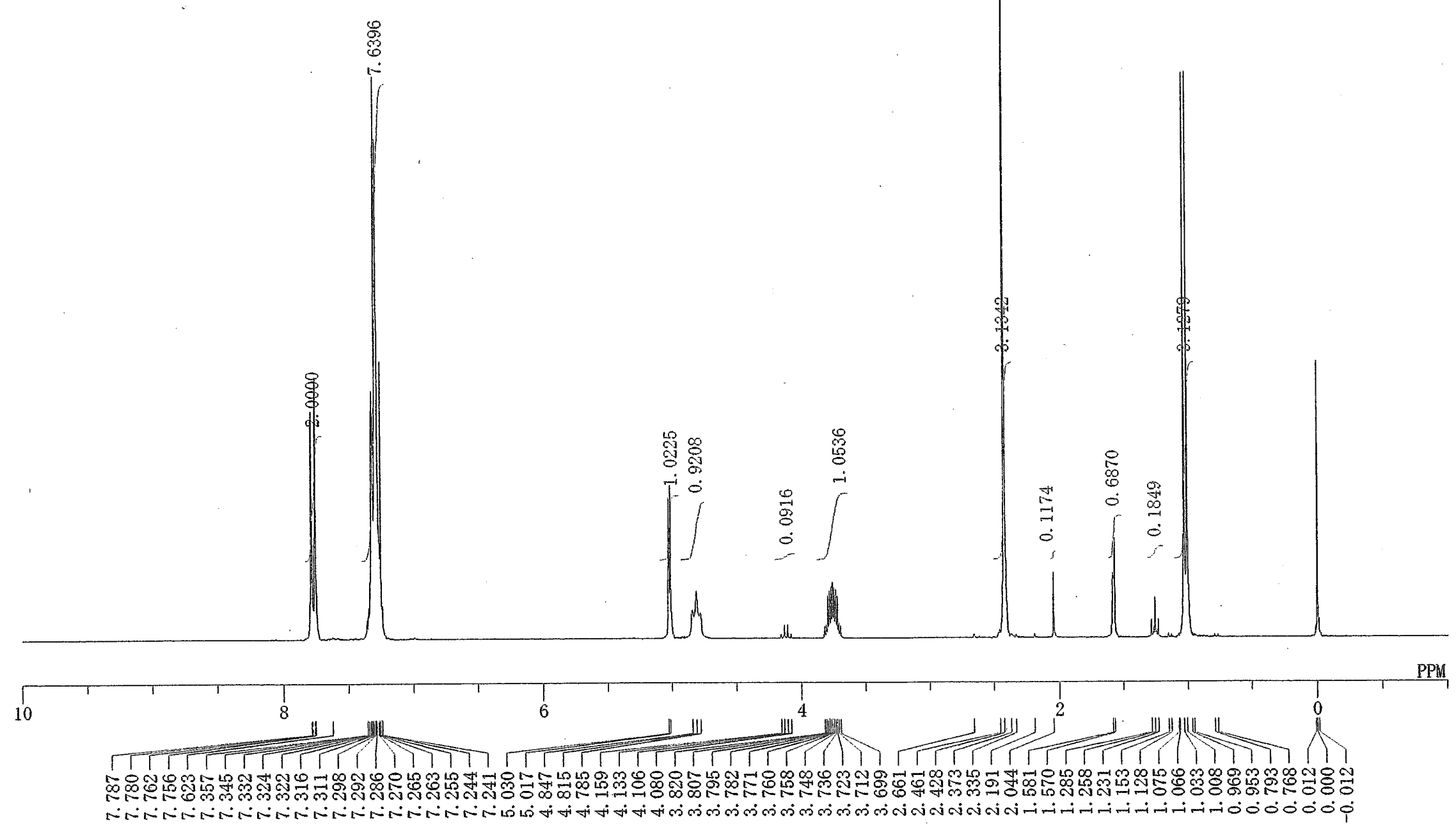

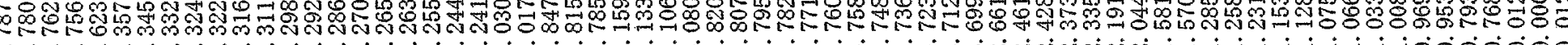

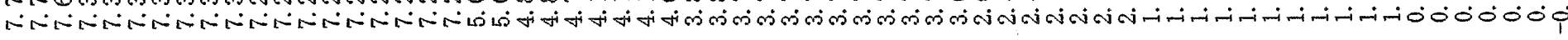




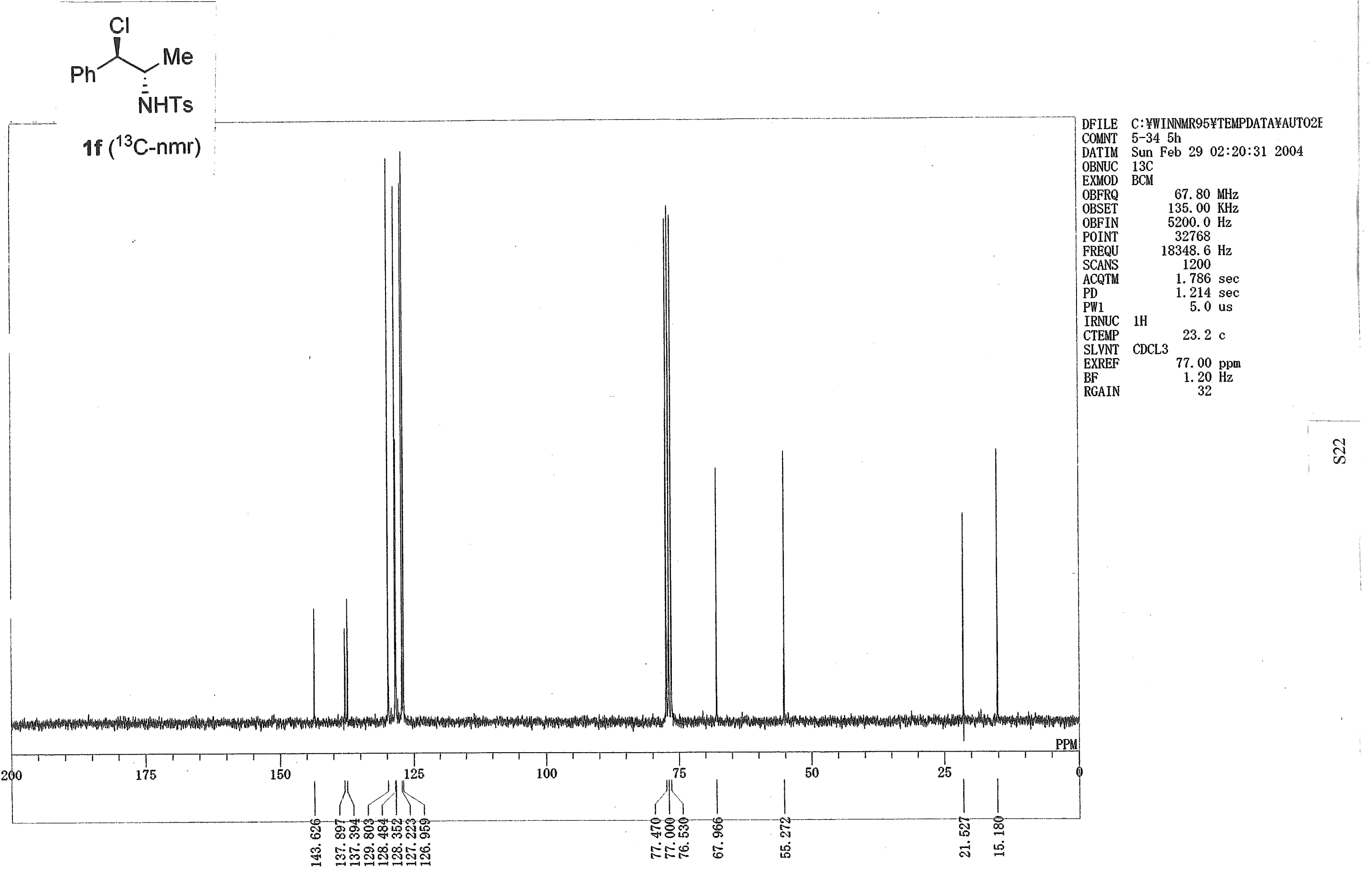




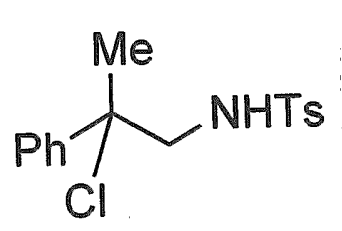

Ih $\left({ }^{1} H-n m r\right)$

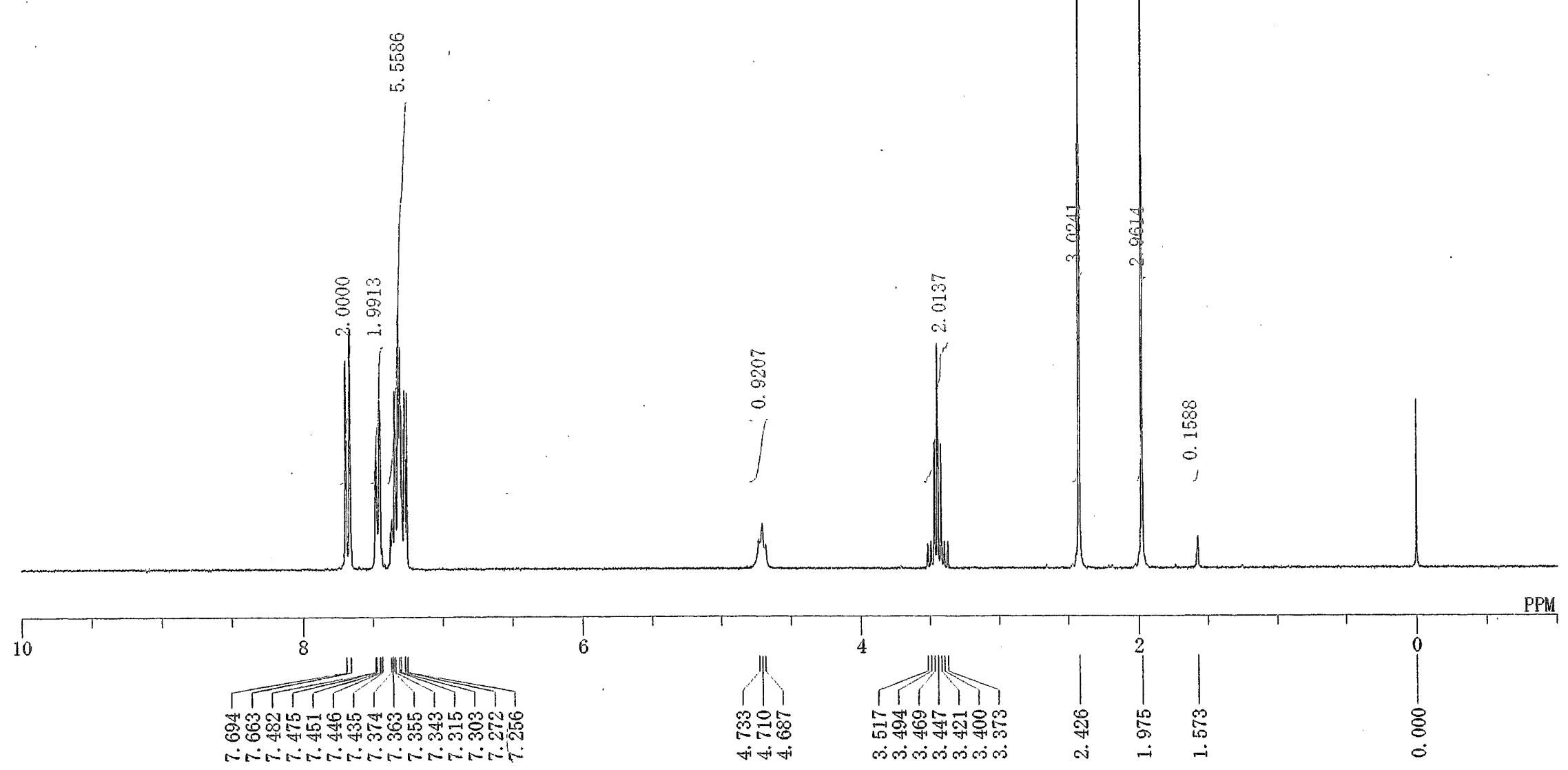




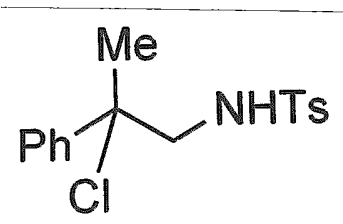

Ih $\left({ }^{13} \mathrm{C}-\mathrm{nmr}\right)$

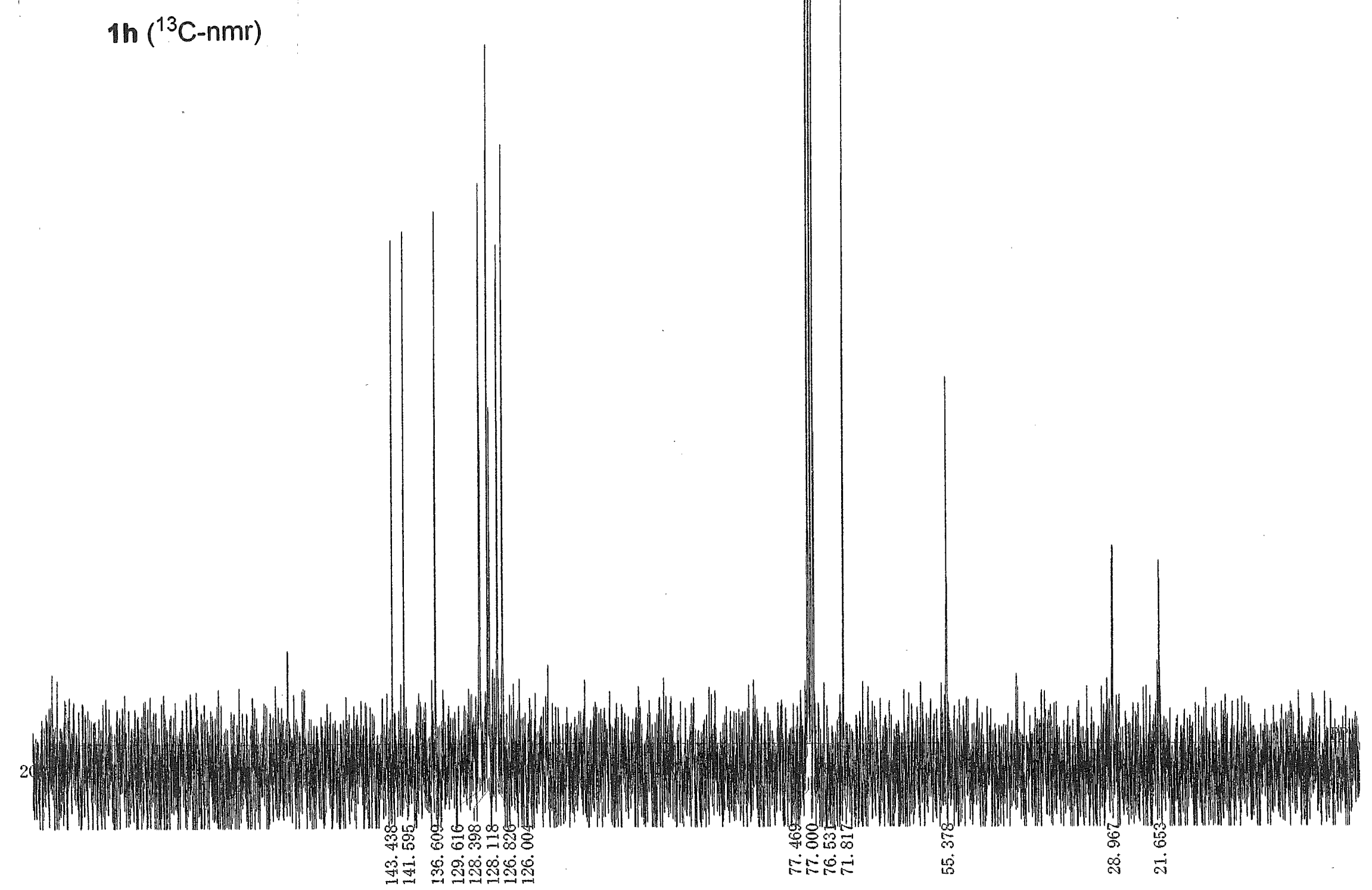




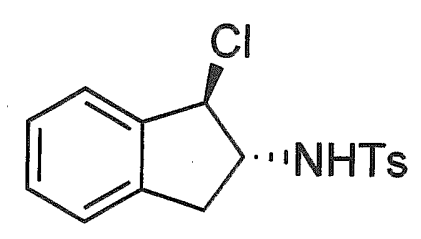

\section{$1 i\left({ }^{1} H-n m r\right)$}
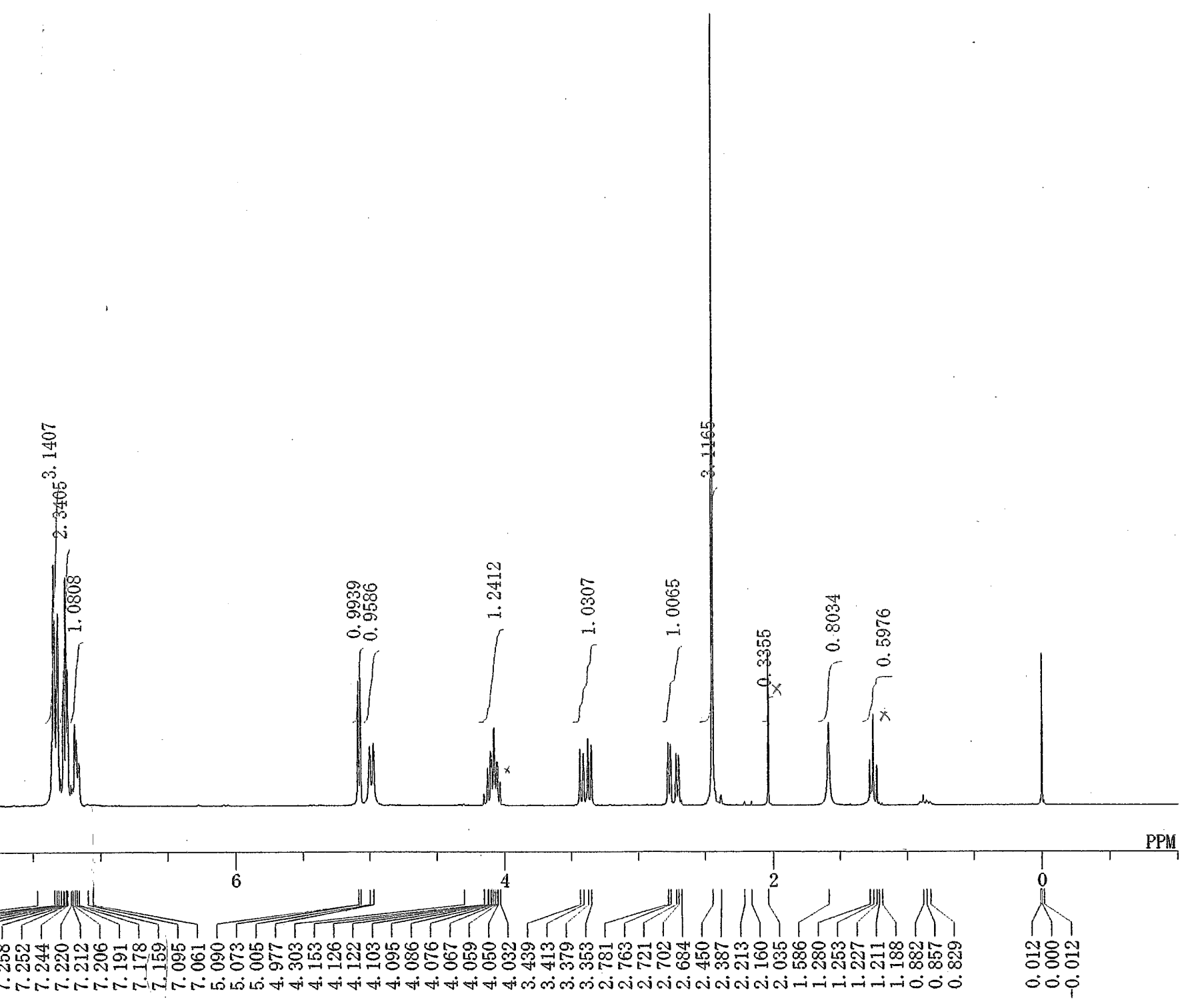
Tा111111117T

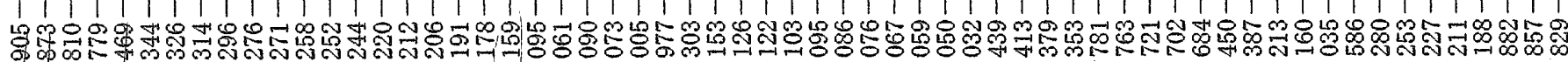

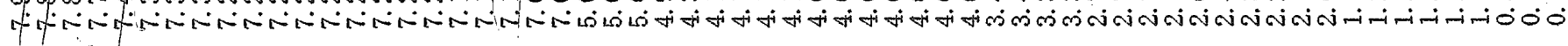




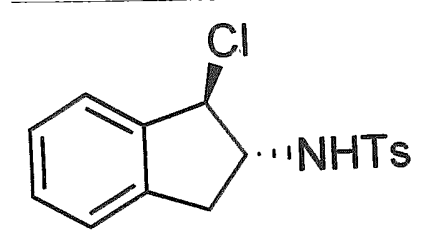

$1 \mathrm{i}\left({ }^{13} \mathrm{C}-\mathrm{nmr}\right)$
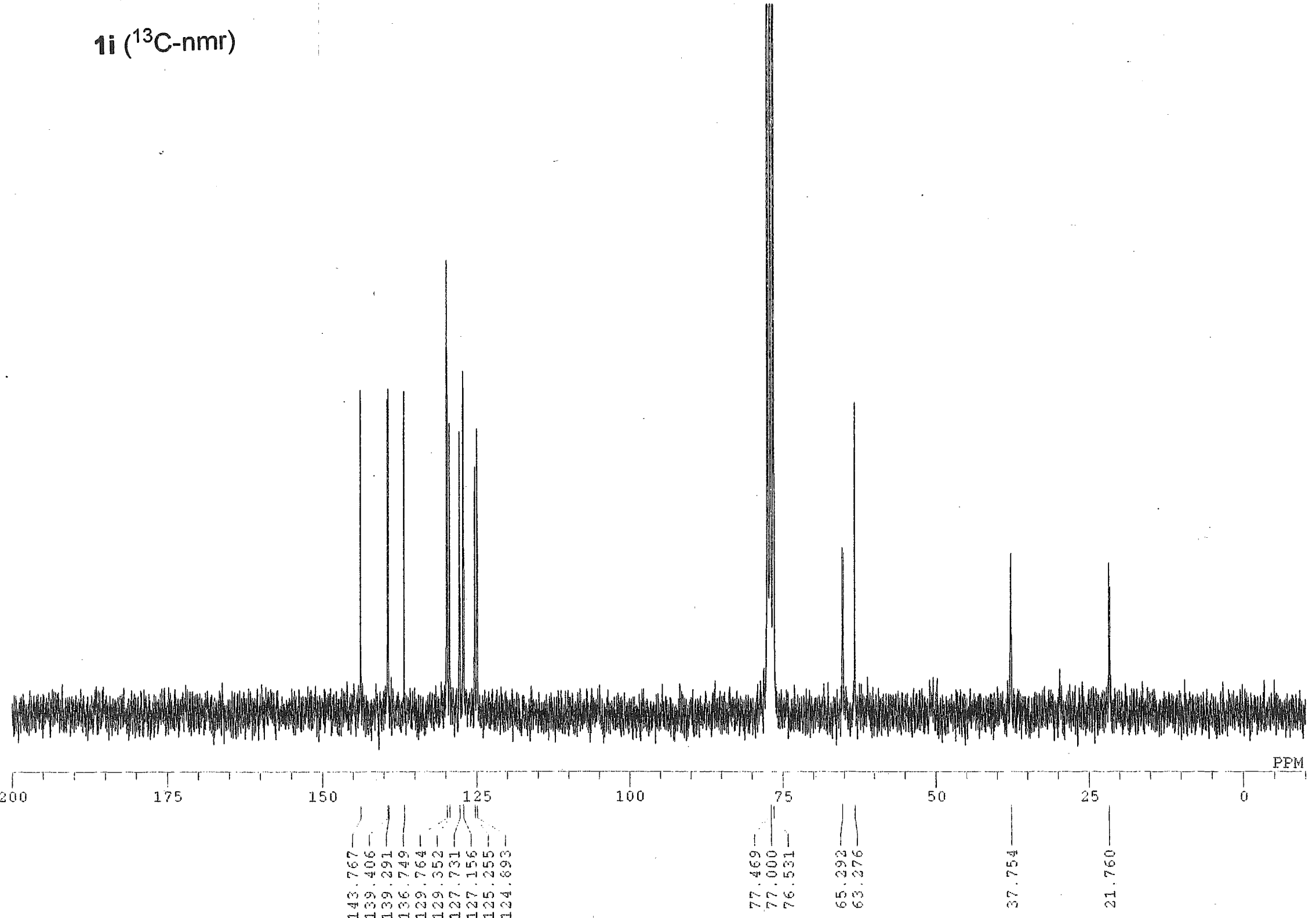

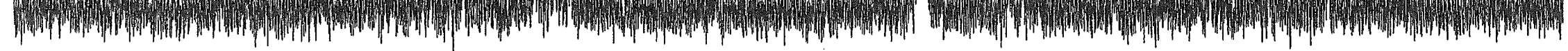

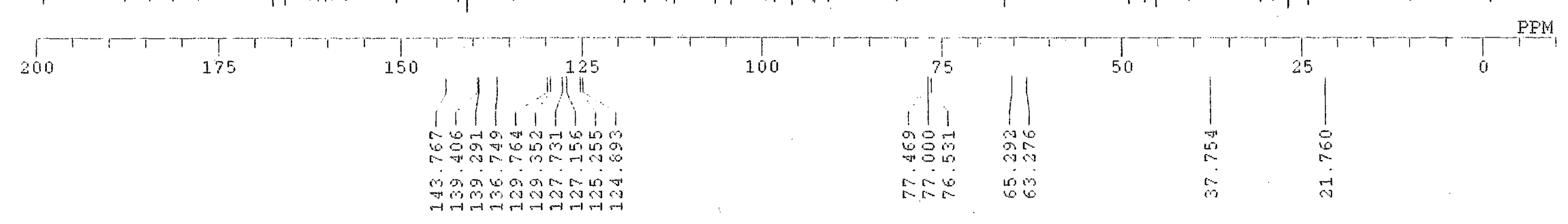



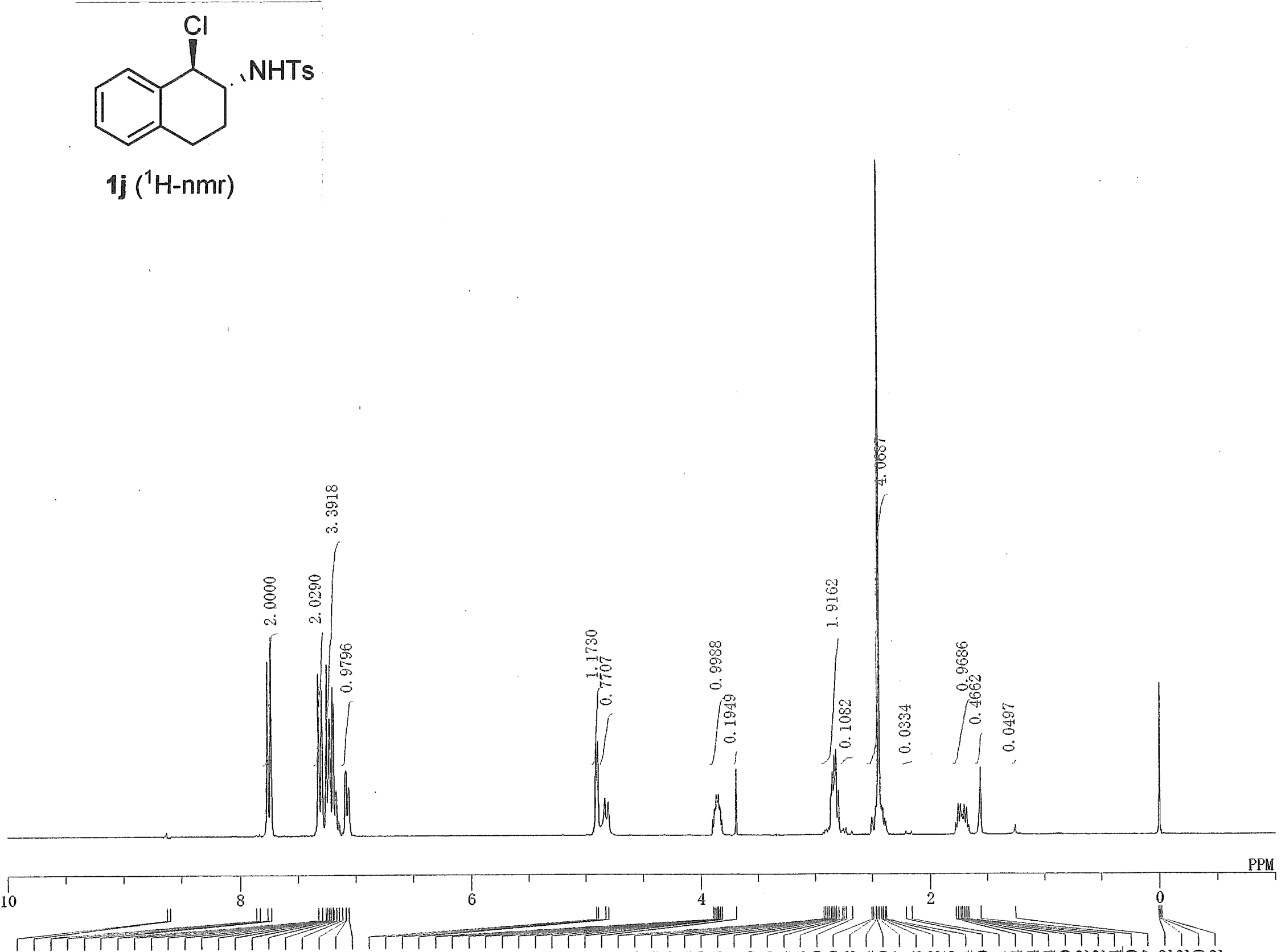

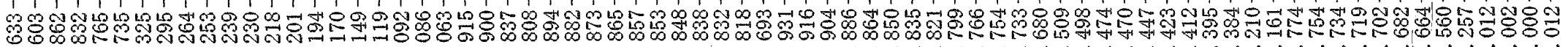

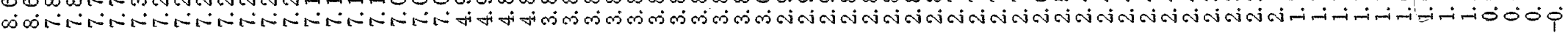




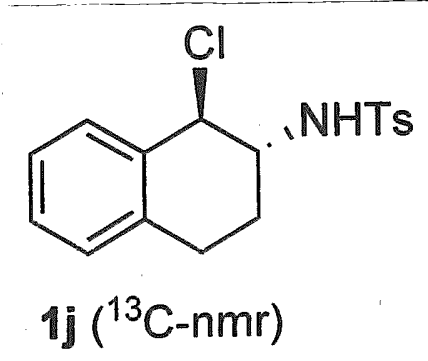

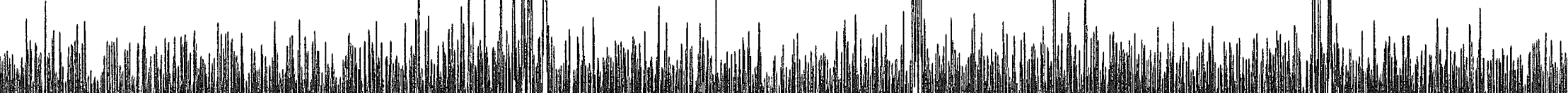
2001

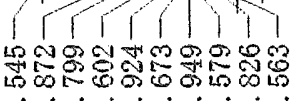

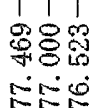
喿 总
8.8
ฆู่ส 


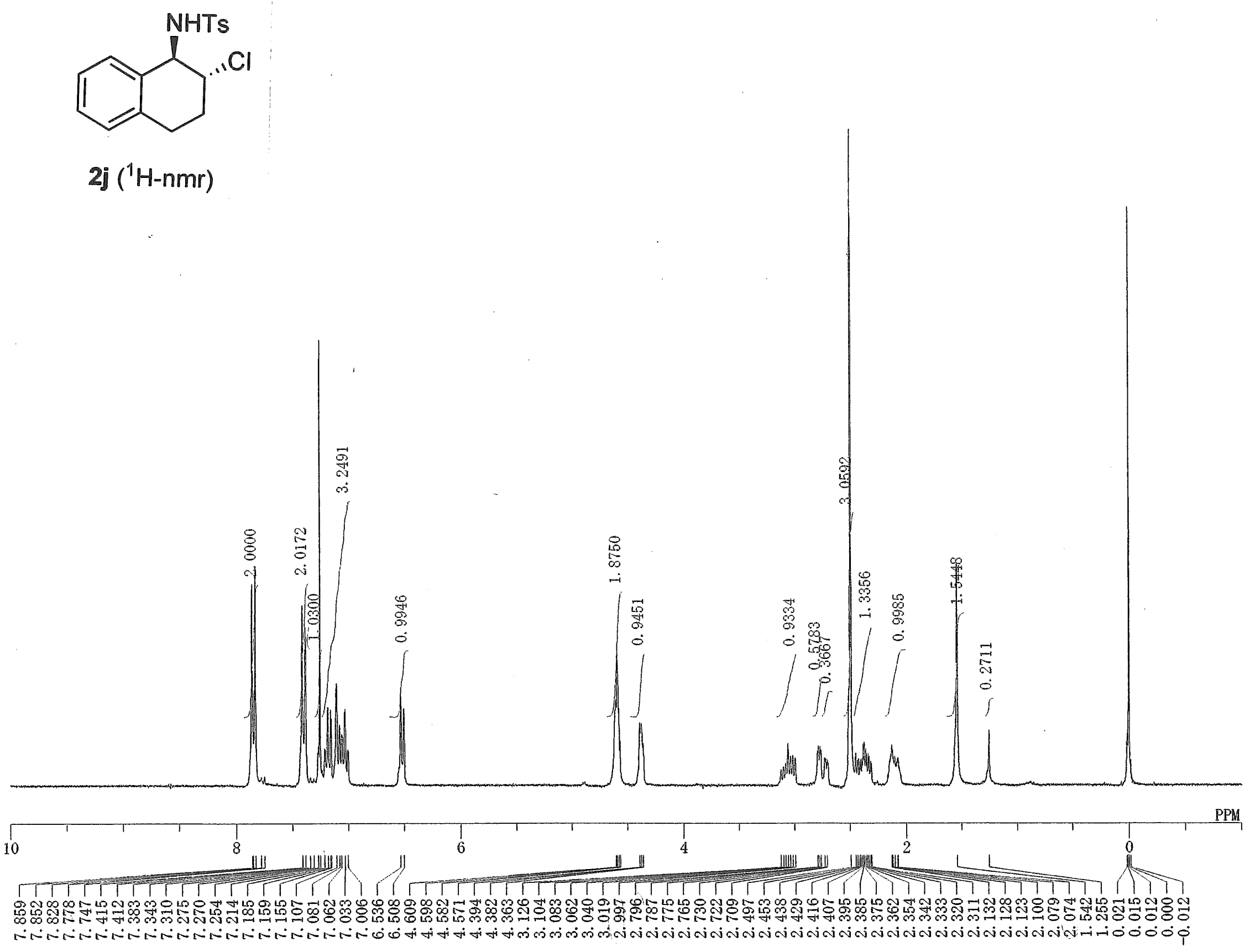




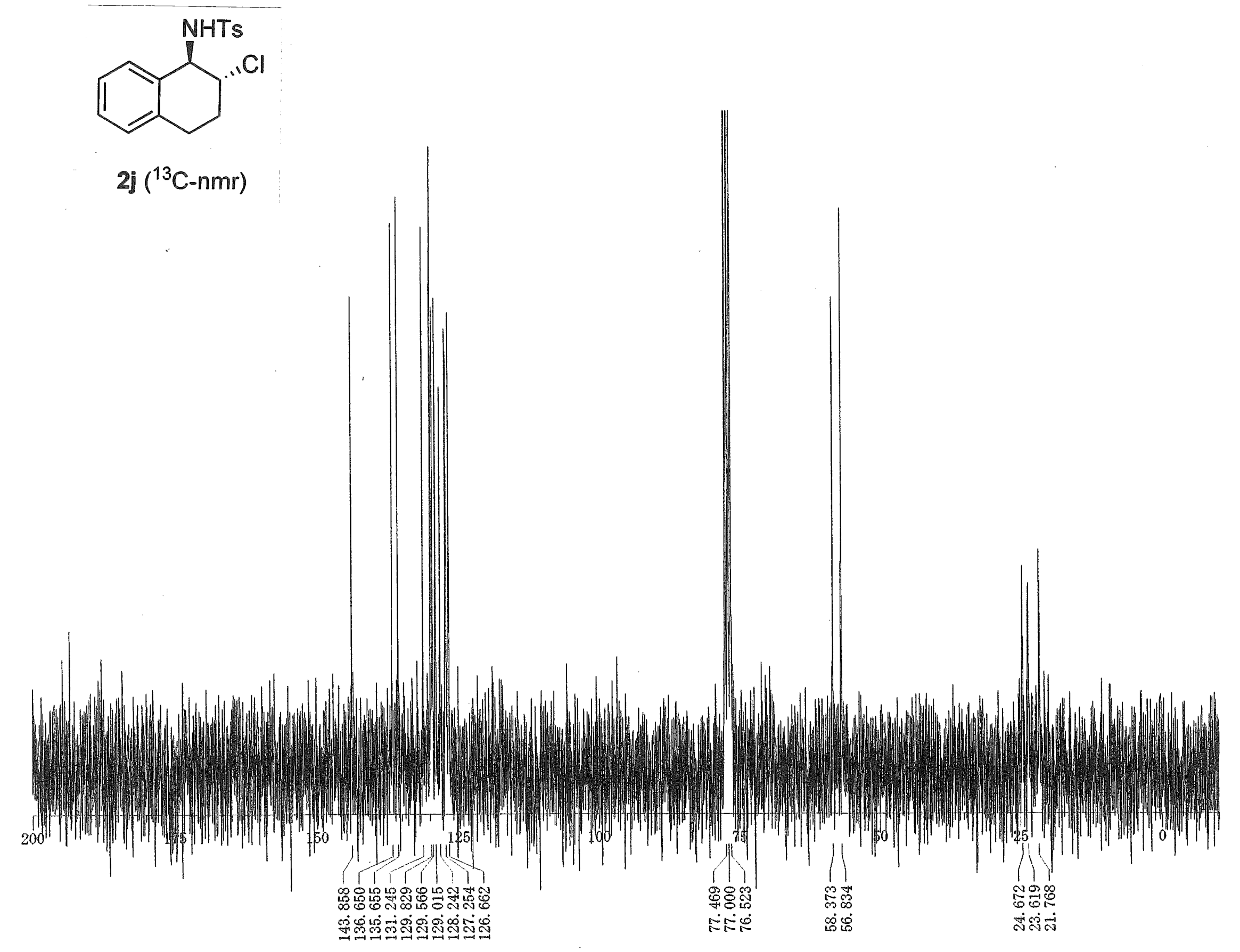



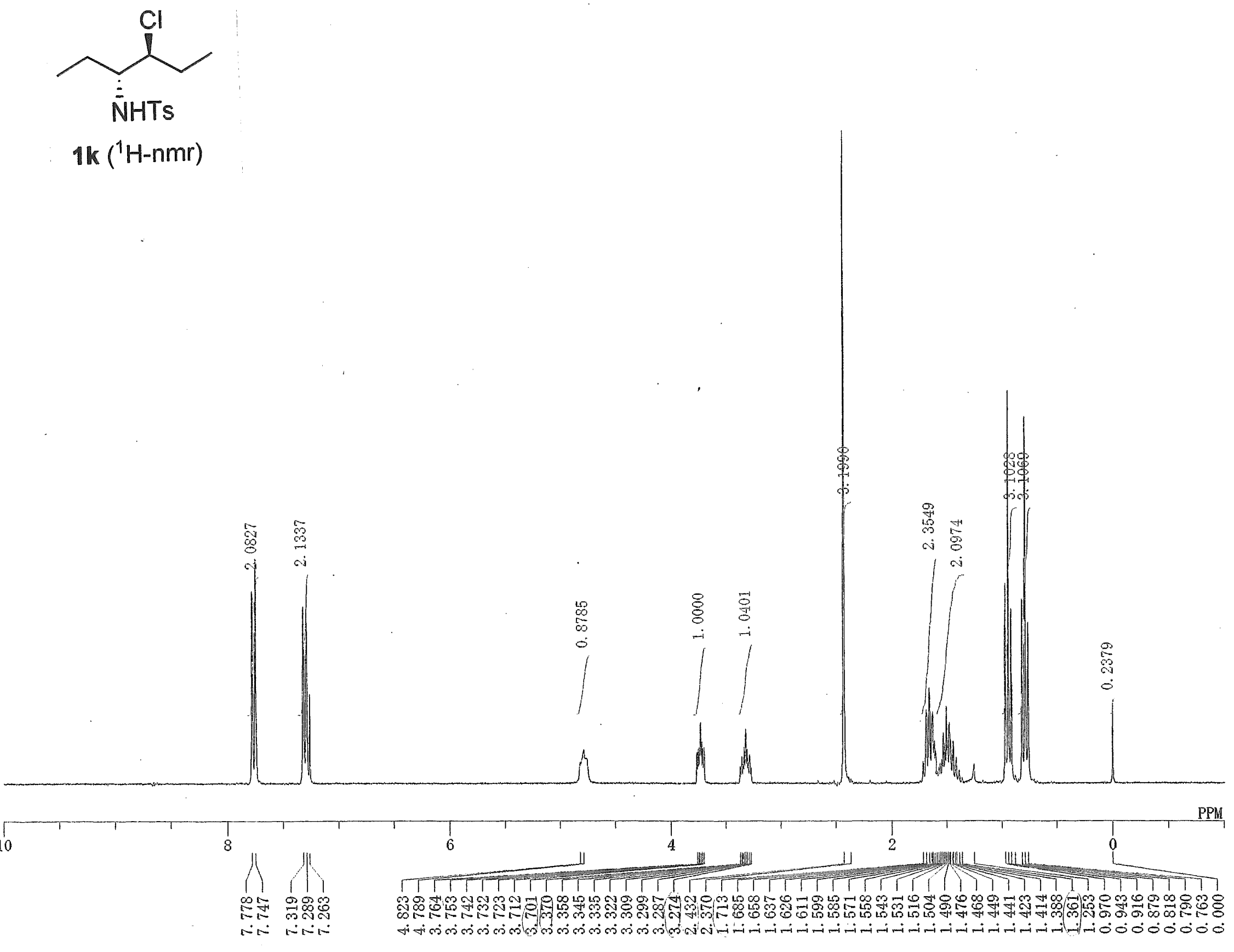


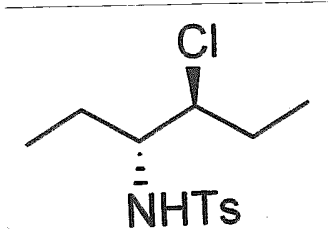

$1 k\left({ }^{13} \mathrm{C}-n m r\right)$

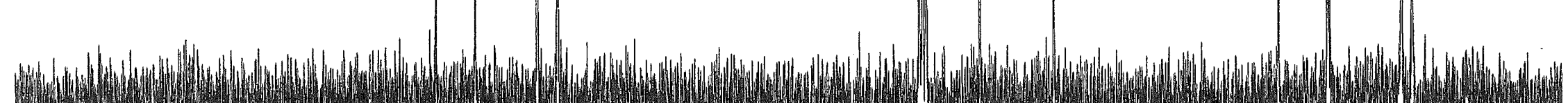

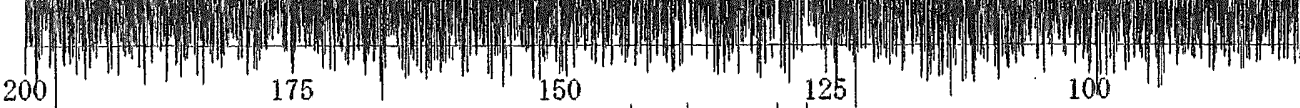
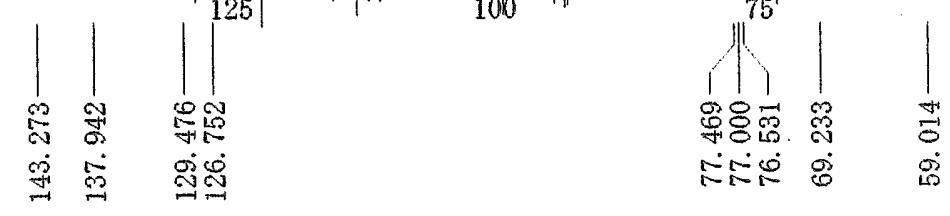

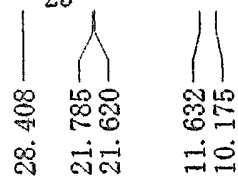




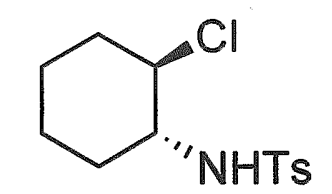

$\mathbb{1}\left({ }^{1} \mathrm{H}-\mathrm{nmr}\right)$

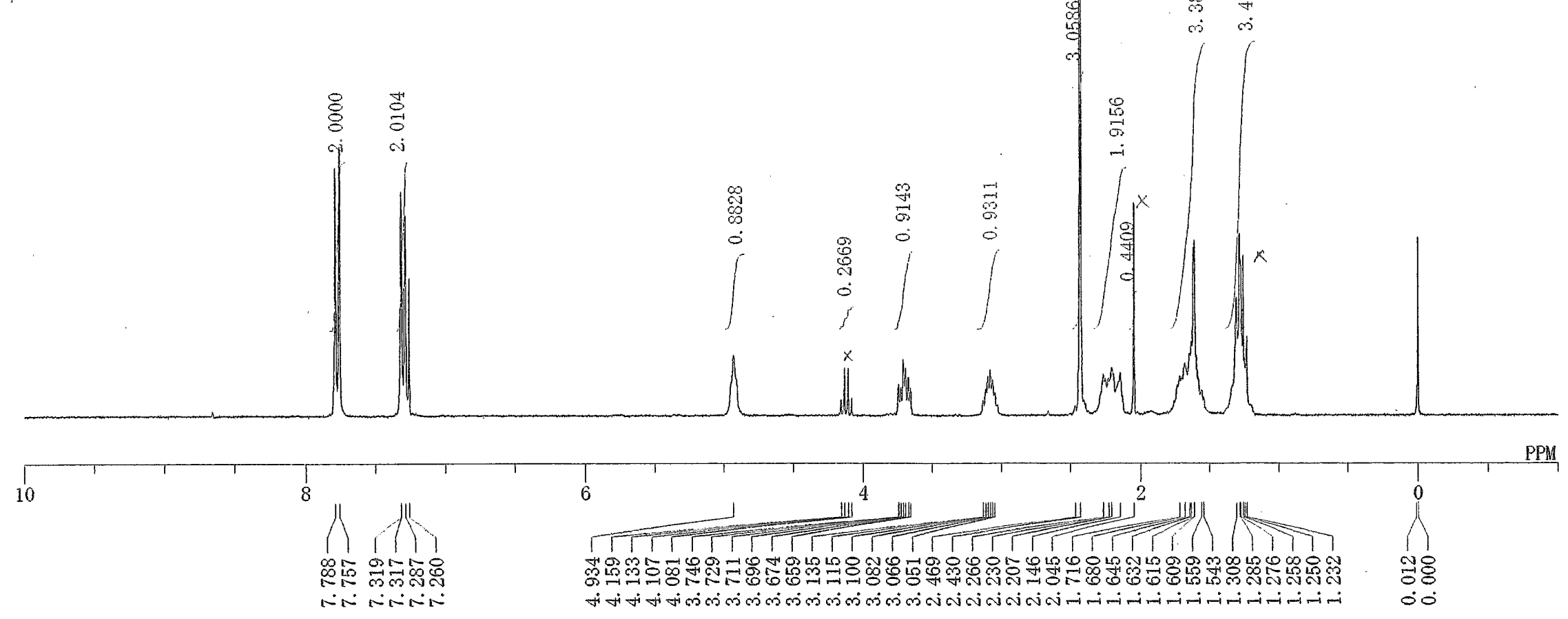




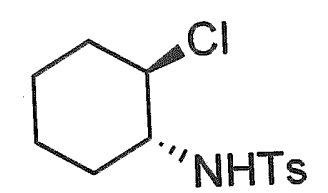

$11\left({ }^{13} \mathrm{C}-n m r\right)$

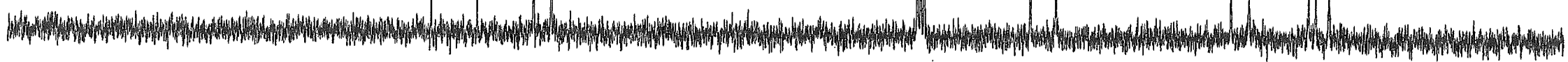

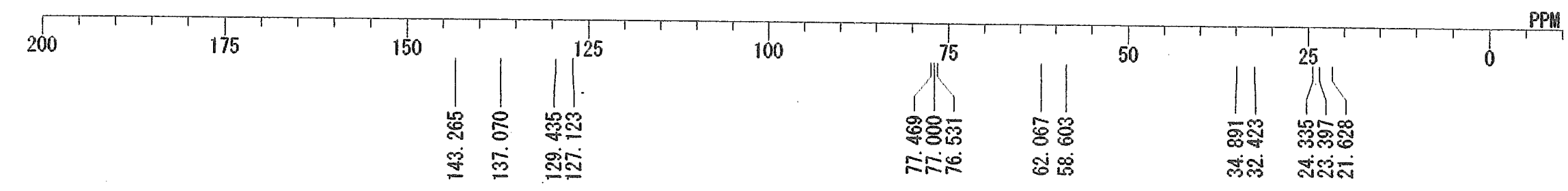




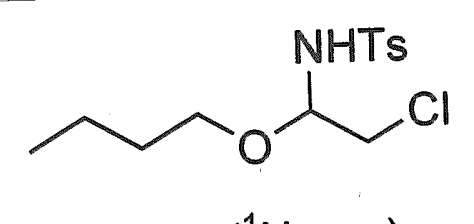

$1 \mathrm{~m}\left({ }^{1} \mathrm{H}-\mathrm{nm} \mathrm{r}\right)$

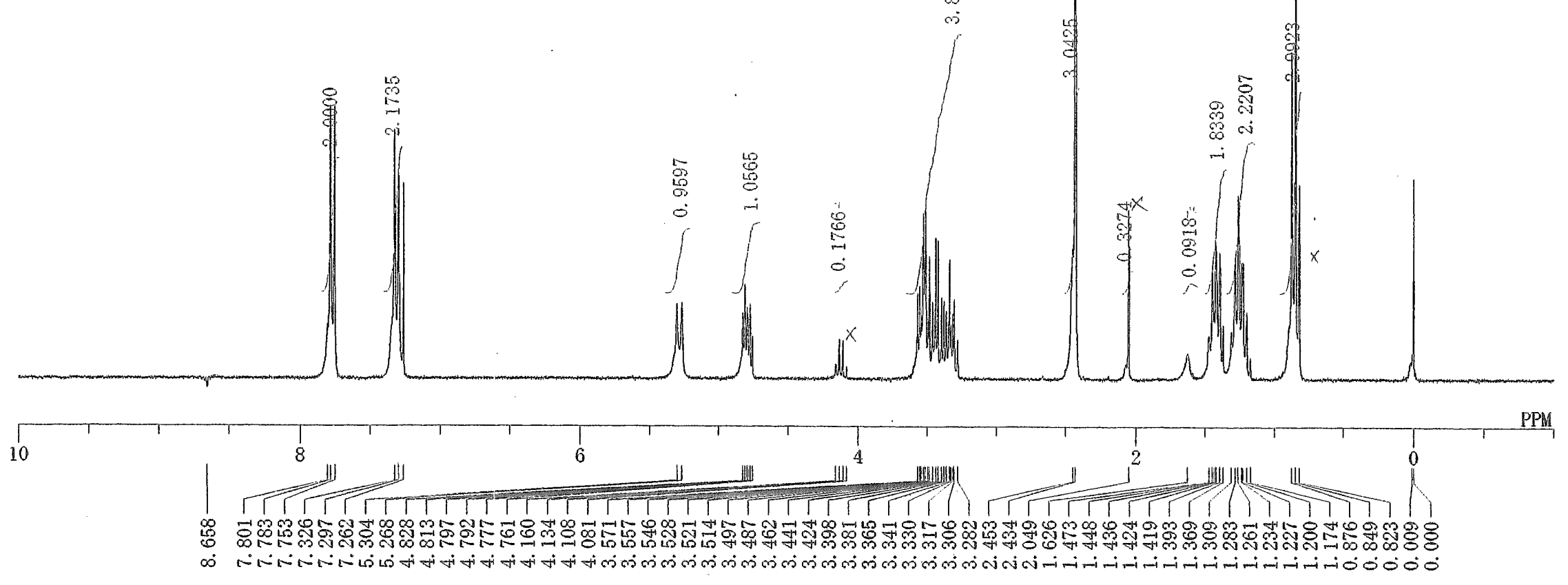



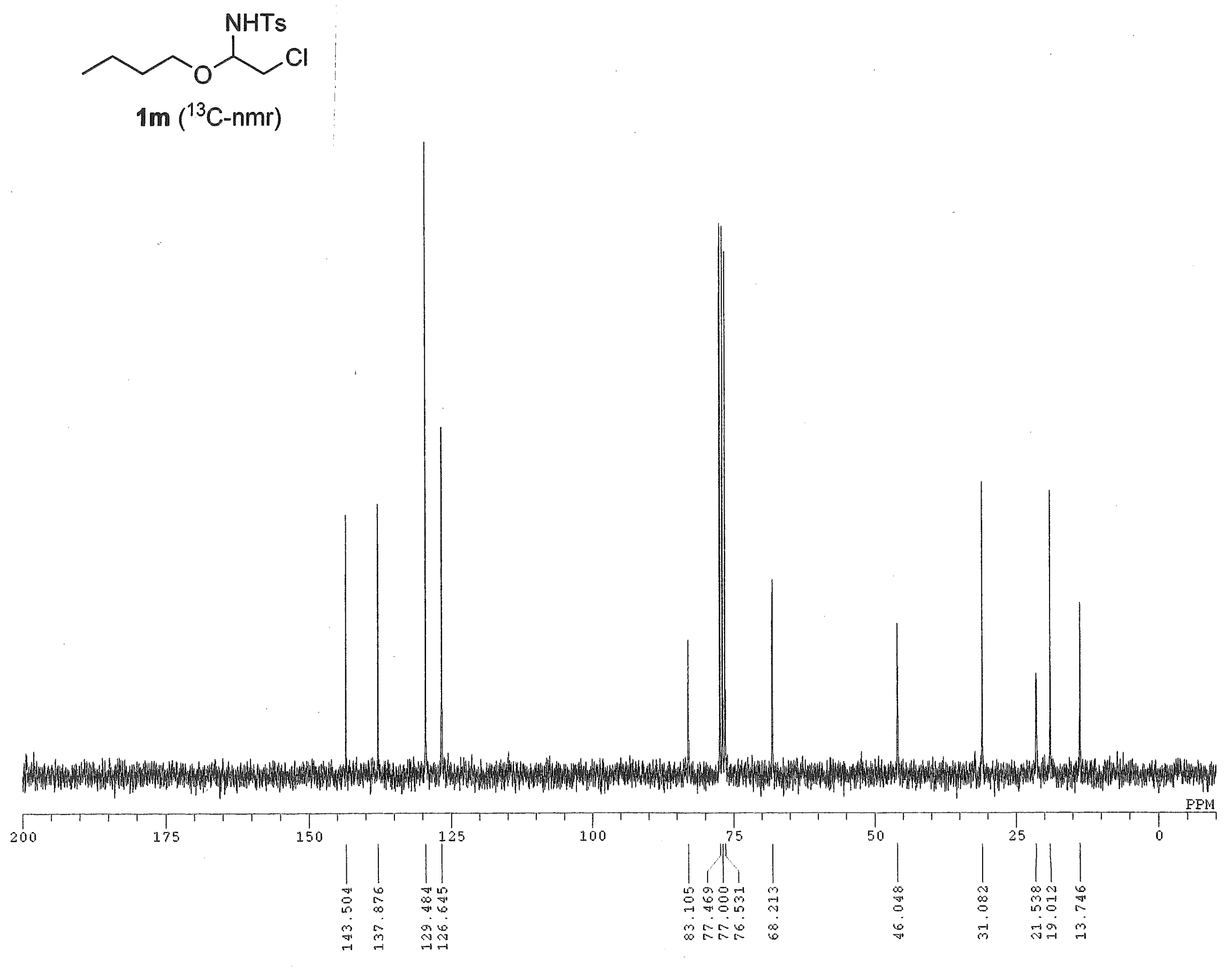

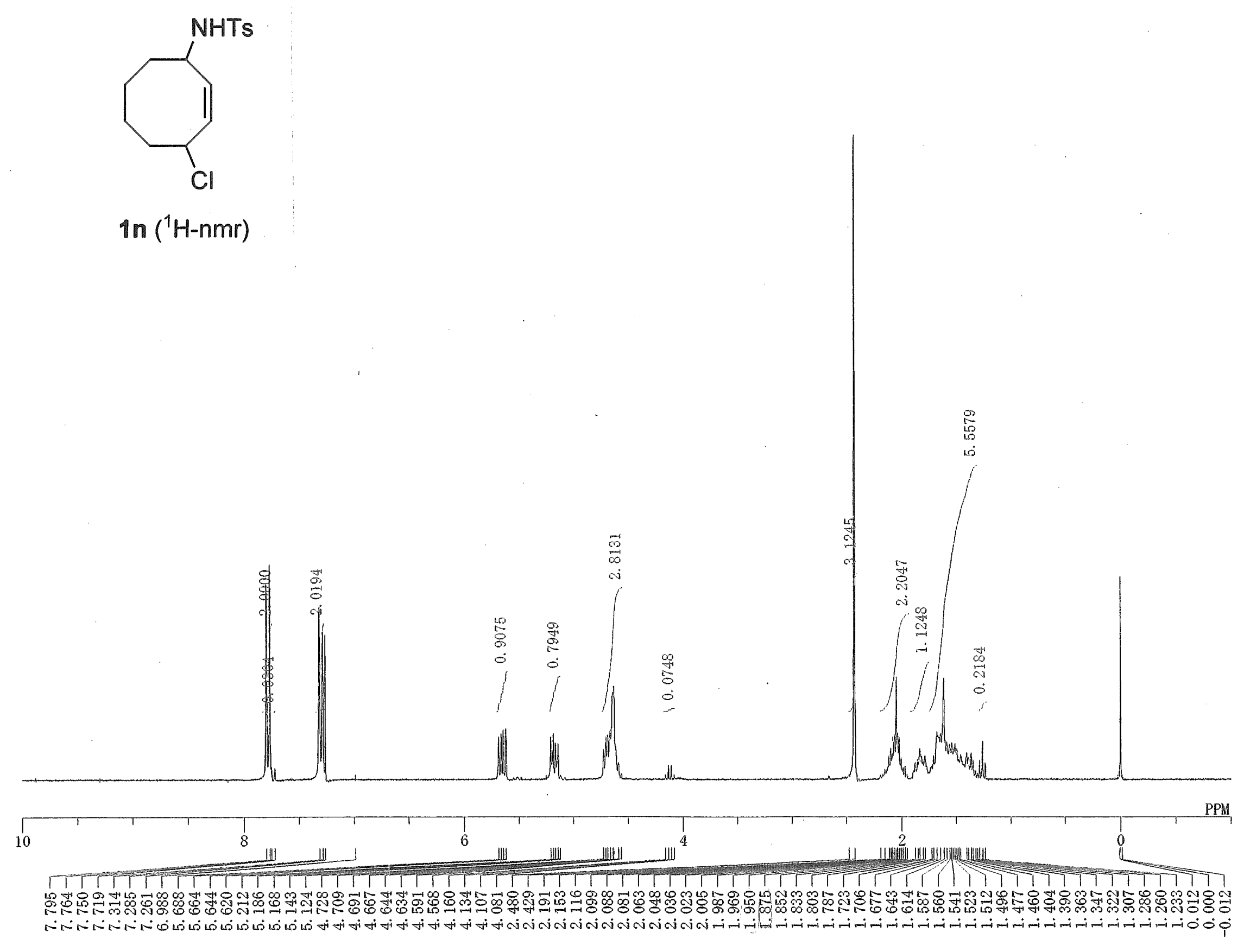


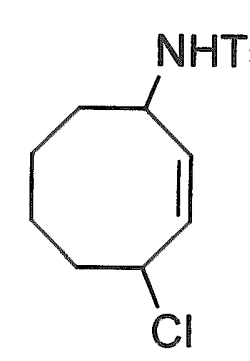

In $\left({ }^{13} \mathrm{C}-\mathrm{nmr}\right)$
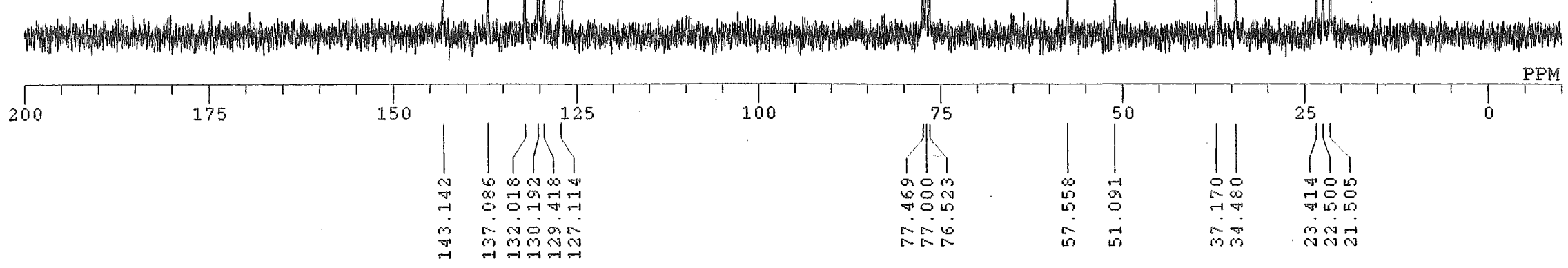